\title{
Two-sided Matching Based Cooperative Spectrum Sharing
}

\author{
Lin Gao, Member, IEEE, Lingjie Duan, Member, IEEE, and Jianwei Huang, Fellow, IEEE
}

\begin{abstract}
Dynamic spectrum access (DSA) can effectively improve the spectrum efficiency and alleviate the spectrum scarcity, by allowing unlicensed secondary users (SUs) to access the licensed spectrum of primary users (PUs) opportunistically. Cooperative spectrum sharing is a new promising paradigm to provide necessary incentives for both PUs and SUs in dynamic spectrum access. The key idea is that SUs relay the traffic of PUs in exchange for the access time on the PUs' licensed spectrum. In this paper, we formulate the cooperative spectrum sharing between multiple PUs and multiple SUs as a two-sided market, and study the market equilibrium under both complete and incomplete information. First, we characterize the sufficient and necessary conditions for the market equilibrium. We analytically show that there may exist multiple market equilibria, among which there is always a unique Paretooptimal equilibrium for PUs (called PU-Optimal-EQ), in which every PU achieves a utility no worse than in any other equilibrium. Then, we show that under complete information, the unique Pareto-optimal equilibrium PU-Optimal-EQ can always be achieved despite the competition among PUs; whereas, under incomplete information, the PU-Optimal-EQ may not be achieved due to the mis-representations of SUs (in reporting their private information). Regarding this, we further study the worse-case equilibrium for PUs, and characterize a Robust equilibrium for PUs (called PU-Robust-EQ), which provides every PU a guaranteed utility under all possible mis-representation behaviors of SUs. Numerical results show that in a typical network where the number of PUs and SUs are different, the performance gap between PU-Optimal-EQ and PU-Robust-EQ is quite small (e.g., less than $10 \%$ in the simulations).
\end{abstract}

Index Terms-Cooperative Spectrum Sharing, Game Theory, Two-Sided Matching, Market Equilibrium

\section{INTRODUCTION}

\subsection{Background and Motivation}

Wireless spectrum is becoming increasingly congested and scarce with the explosive development of wireless devices and services. Dynamic spectrum access (DSA) is a promising approach to increase the spectrum efficiency and alleviate the spectrum scarcity, by allowing unlicensed secondary users (SUs) to opportunistically access to the spectrum licensed to primary users (PUs) [1]-[4]. To successfully implement DSA, it is important to offer necessary incentives for PUs to open their spectrum for SUs' utilizations, and for SUs to access the PUs' spectrum despite of the potential costs [5]-[7].

Cooperative spectrum sharing (also called cooperative spectrum leasing [8]) has been proposed as an effective approach to offer crucial incentives for both PUs and SUs in DSA [8]-[14]. The basic idea is that SUs relay the traffic of PUs in exchange for the opportunities to access the PUs' licensed spectrum. With the cooperative spectrum sharing, PUs can increase their transmission rates through the cooperative relay of SUs, and SUs can

- Lin Gao is with the School of Electronic and Information Engineering, Harbin Institute of Technology Shenzhen Graduate School, China, Email: gaolin@hitsz.edu.cn; Lingiie Duan is with Engineering Systems and Design Pillar, Singapore University of Technology and Design (SUTD), Singapore, Email: lingjie_duan@sutd.edu.sg; Jianwei Huang (corresponding author) is with the Network Communications and Economics Lab, Department of Information Engineering, The Chinese University of Hong Kong, Email: jwhuang@ie.cuhk.edu.hk.

- This work is supported by the General Research Funds (Project Number CUHK 412713) established under the University Grant Committee of the Hong Kong Special Administrative Region, China.

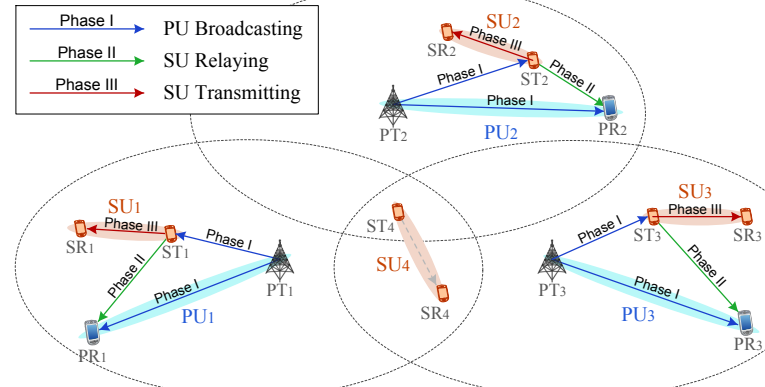

Fig. 1. Illustration of cooperative spectrum sharing.

obtain transmission opportunities on the PUs' licensed spectrum. Thus, it will lead to a win-win situation for PUs and SUs ${ }_{1}^{1}$ Figure 1 illustrates an example of cooperative spectrum sharing between 3 PUs and 4 SUs, ${ }^{2}$ where each SU $i$ cooperates with PU $i$ for $i \in\{1,2,3\}$, and SU 4 does not cooperate with any PU. SUs relay traffic for intended PUs according to a pre-defined cooperative protocol, such as amplified-and forward (AF) and decode-and-forward (DF). Hence, each cooperation frame is divided into 3 phases: in Phase I, each PU $i^{\prime}$ s transmitter $\left(\mathrm{PT}_{i}\right)$ broadcasts its messages to its receiver $\left(\mathrm{PR}_{i}\right)$ as well as to the cooperating $\mathrm{SU} i$; in Phase II, each SU $i$ 's transmitter $\left(\mathrm{ST}_{i}\right)$ forwards the received PU $i$ 's signal to the PU $i$ 's receiver $\mathrm{PR}_{i}$; and in Phase III, each SU $i$ transmits its own messages (from $\mathrm{ST}_{i}$ to $\mathrm{SR}_{i}$ )

1. Cooperative spectrum sharing can also be viewed as an enhanced cooperative relay scheme. From the perspective of cooperative relays (e.g., D2D relays in 5G system [28]), it can provide the necessary incentives for SUs to relay the traffic of PUs.

2. Here each PU $i$ is a dedicated transceiver pair $\left\{\mathrm{PT}_{i}, \mathrm{PR}_{i}\right\}$, and each $\mathrm{SU} i$ is also a dedicated transceiver pair $\left\{\mathrm{ST}_{i}, \mathrm{SR}_{i}\right\}$. 
on the PU $i$ 's spectrum.

While there are some prior works considering the cooperative spectrum sharing problem [8]-[14], all of these works focused on the interactions between one PU and one or multiple SUs. Our work advances the research in this area by analyzing the interaction between multiple PUs and multiple SUs as shown in Figure 11. This more practical scenario is significantly more challenging to analyze, as not only SUs compete with each other for the PUs' licensed spectrums, but PUs also compete with each other for the SUs' collaborations. An important question arising in such a multi-PU multi-SU scenario is:

- Which PU cooperates with which SU, and what is the resource exchange (i.e., the PU's spectrum access time reward and the SU's relay effort) between them?

As the traditional matching problem usually studies the binary pairing of users without considering the resource exchange details, our problem is actually an extended matching problem.

\subsection{Solution and Contribution}

In this work, we formulate the cooperative spectrum sharing problem between multiple PUs and multiple SUs as a two-sided matching market, with PUs as one side and SUs as the other side. A PU is matched to an SU means that the PU cooperates with the SU under certain resource exchange agreement. Accordingly, a matching between PUs and SUs defines not only their collaborative relationships, but also the resource exchange between each pair of matched PU and SU. An equilibrium is defined as a stable matching, from which neither PUs nor SUs have the incentive to deviate. For such a two-sided market, we want to answer the following questions:

- what is the market equilibrium, and which equilibrium will emerge in different information scenarios?

Note that both problems are challenging due to the following reasons. First, finding the stable matching of a two-sided matching market is well-known an NP-hard problem, and thus mathematically intractable. Second, in some two-sided matching markets including the one in this paper, there may be an infinite number of market equilibria, and analytically characterizing these market equilibria is challenging. Third, different information scenarios (depending on how much information that PUs know) may lead to different market equilibria. Characterizing the equilibrium in different information scenarios is also a challenging problem.

We will study the market equilibrium in two different information scenarios systematically: complete and incomplete information. In the former case, each PU knows the whole network information, while in the latter case, each PU knows only its local network information (see Section 3.F for details). To the best of our knowledge, this is the first paper that systematically studies cooperative spectrum sharing between multiple PUs and multiple SUs by using the matching theory. The main results and contributions of this paper are summarized as follows.
- Two-sided Market Model and Solution Technique: We formulate the cooperative spectrum sharing between multiple PUs and multiple SUs as a two-sided matching market, and comprehensively study the market equilibrium (stable matching) in both complete and incomplete information scenarios.

- Equilibrium Analysis: We characterize the sufficient and necessary conditions for market equilibrium, and show that there may be an infinite number of market equilibria. We further show that these is always a unique Pareto-optimal equilibrium for PUs (PU-Optimal-EQ), in which every PU achieves a utility no worse than that in any other equilibrium.

- Complete and Incomplete Information: We show that under complete information, the unique Paretooptimal equilibrium for PUs (PU-Optimal-EQ) can always be achieved despite of the competition among PUs. Under incomplete information, however, the PU-Optimal-EQ may not be achieved due to the mis-representations of SUs. To this end, we characterize a Robust equilibrium for PUs (PU-RobustEQ), which provides every PU a guaranteed utility under all possible mis-representation behaviors of SUs.

- Performance Evaluation: Numerical studies show that each PU's utility increases (or decreases) with the number of SUs (or PUs) in both PU-Optimal$E Q$ and PU-Robust-EQ. In many practical network scenarios where the numbers of PUs and SUs are often different, the performance gap between PUOptimal-EQ and PU-Robust-EQ is quite small (e.g., less than $10 \%$ in the simulations). This implies that the equilibrium performance depends more on the market structure than on the information scenario.

The rest of this paper is organized as follows. In Section 2, we review the related literature. In Section 3. we present the system model. In Section 4 , we provide the two-sided market formulation. In Section 5, we use a simplified example to illustrate the equilibrium. In Section 6. we study the equilibria of a general model. In Section 7, we show which equilibria will emerge in different information scenarios. We provide numerical results in Section 8 , and finally conclude in Section 9.

\section{Literature Review}

\section{A. Cooperative Spectrum Sharing}

Most prior work on cooperative spectrum sharing focused on the interactions between one PU and one or multiple SUs [8]-[14]. Some assumed that the PU has complete information of SUs (e.g., SUs' relay channel gains and sensitivities to power consumption) [8]-[11], while others considered that the PU has limited information about SUs [12], [13]. These works used either Stackelberg game or contract theory to model and analyze the problem. However, both approaches are difficult to be extended to the scenario of multiple PUs and multiple SUs. A closely related paper that considers the 
interactions between multiple PUs and multiple SUs is [14], where authors proposed a distributed algorithm to reach a stable matching that is weak Pareto optimal. Our work generalizes the result of [14] in the following way: (i) we analytically show that there are multiple (possibly infinite) stable matchings including the Pareto optimal one studied in [14], (ii) we characterize the necessary and sufficient conditions for all possible stable matchings, and (iii) we propose distributed algorithms converging to different stable matchings, given different information available to PUs.

\section{B. Two-sided Matching Market}

In economics, two-sided matching market is an effective framework and widely-used for studying the interactions between two disjoint player sets in a two-sided market setting. In such markets, the matching theory can systematically capture not only the cooperative interactions between users in different sides, but also the competitive interactions between users on the same side. Most early results in this area focused on the matching under complete information, without considering the incentive issues under incomplete information. The first basic two-sided market models were proposed by Gale and Shapley [15]. Shapley and Shubik [16] and Thompson [17] studied the more general models with additive and transferable utilities. Crawford and Knoer [18], [19] studied the two-sided labor models with nontransferable utilities. Some later results [20], [21] studied the incentive issue under incomplete information. Our work differs from the above works: we consider not only the binary matching decision, but also the detailed resource exchange between each pair of matched users.

It is notable that our analysis techniques and engineering insights are applicable to other wireless network problems that can be modeled as a two-sided market. The equilibrium obtained in this paper provides a quite general characterization of the network stable outcome in a wide range of large complicated networks.

\section{System MOdel}

We consider a DSA network with a set $\mathcal{M} \triangleq\{1, \ldots, M\}$ of PUs and a set $\mathcal{N} \triangleq\{1, \ldots, N\}$ of SUs. Each PU $m \in \mathcal{M}$ (or SU $n \in \mathcal{N})$ is a dedicated transceiver pair $\left\{\mathrm{PT}_{m}, \mathrm{PR}_{m}\right\}$ (or $\left\{\mathrm{ST}_{n}, \mathrm{SR}_{n}\right\}$ ) as illustrated in Figure 1. A PU has the exclusive usage right of a licensed frequency band (with a normalized bandwidth). We assume that the frequency bands of different PUs are non-overlapping, and thus there is no interference among PUs' transmissions. As in many existing literature [8]-[14], we further assume that there exists a common control channel for the communications and interactions between PUs and SUs.

On one hand, PUs may suffer from low transmission rates or high outage probabilities due to the poor channel conditions between their transmitters and receivers caused by, for example, the long-distance attenuation, shadowing, and fading. Thus, PUs want to employ

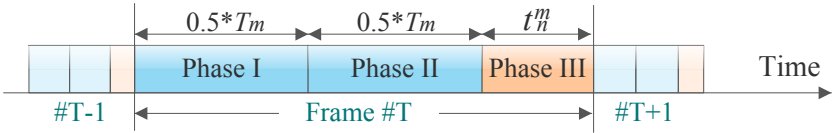

Fig. 2. Frame structure of cooperative spectrum sharing.

SUs as relays to improve their transmission rates. On the other hand, SUs need spectrum resources for their own transmissions, but cannot access the PUs' licensed spectrum without PUs' permissions. Thus, we expect a win-win situation where SUs relay PUs' traffic in order to get free access time on the PUs' spectrums.

To facilitate the practical implementation, we assume that one PU can choose at most one SU to relay its traffic at a particular time (but can change the relaying $\mathrm{SU}$ at different time). This is motivated by existing results that choosing the most appropriate relay is usually sufficient to achieve the optimal (or close-to-optimal) performance [22]. Moreover, this can be implemented more easily in practice, as there is no need to consider the coordination among multiple relays. We further assume that each SU can serve at most one PU at a particular time (but can change the serving PU at different time). Therefore, a key question arising in such a scenario is: who will relay whose traffic, and how?

\subsection{Cooperative Spectrum Sharing Protocol}

We first consider the cooperative spectrum sharing protocol between a particular PU $m$ and SU $n$ (assuming SU $n$ cooperates with PU $m$ ). As shown in Figure 1, each cooperation period (frame) includes 3 phases: Phases I and II for the cooperative communication between PU $m$ and SU $n$ (each with a fixed period of $T_{m} / 2$ ) ${ }^{3}$ and Phase III for SU n's own transmission (with a period of $\left.t_{n}^{m}\right)$. Thus, the total length of one cooperation frame is $T_{m}+t_{n}^{m}$. For clarity, we illustrate the structure of such a 3-phase transmission frame in Figure 2.

Obviously, the cooperative communication protocol used in Phases I and II plays an important role in the cooperative spectrum sharing. In this paper, we adopt the widely-used Amplified-and-Forward (AF) protocol [23] in Phases I and II ${ }^{4}$ For the analytical convenience, we assume the $A W G N$ channel in the physical links. Note that the channel model can be easily extended to fast fading channels (e.g., Rayleigh channel), by considering the average transmission rate or average outage probability. We further assume that each pair of transmitter and receiver know the channel gain between them through, for example, measuring the received signal strength.

For convenience, we list the key notations in Table 1 . where $m$ is the PU index and $n$ is the SU index. The meaning of each notation will be explained later.

3. The total cooperative transmission period $T_{m}$ is determined by the physical layer specifications. Different PUs may have different $T_{m}$.

4. Note that our analysis can be easily applied to other cooperative protocols (e.g., Decode-and-Forward and Compress-and-Forward) with minor modifications on the utility functions of PUs and SUs. 
TABLE 1

Key Notations

Similarly, the SU $n$ 's transmission rate in Phase III on the PU $m^{\prime}$ s frequency band is given by [23]:

$$
R_{n(m)} \triangleq \log \left(1+\frac{G_{n(m)}^{2}}{\sigma^{2}}\right),
$$

where $G_{n(m)}$ is the SU n's direct channel $\left(\mathrm{ST}_{n}\right.$ to $\left.\mathrm{SR}_{n}\right)$ gain on the PU $m$ 's frequency band. Note that each SU $n$ may have different direct channel gains $G_{n(m)}$ on different PU $m$ 's band, due to the frequency selective fading. Moreover, there is no interference between SUs' transmissions as they operate on non-overlapping bands.

Since the SU $n$ 's transmission occupies $\frac{t_{n}^{m}}{T_{m}+t_{n}^{m}}$ fraction of each frame, its equivalent transmission rate during the entire frame (when cooperating with PU $m$ ) is

$$
\widetilde{R}_{n(m)}=\frac{t_{n}^{m}}{T_{m}+t_{n}^{m}} \cdot \log \left(1+\frac{G_{n(m)}^{2}}{\sigma^{2}}\right) .
$$

We now define the PU's and SU's utilities achieved in the cooperative spectrum sharing. Suppose SU $n$ cooperates with PU $m$ in the following way: SU $n$ relays the PU $m^{\prime}$ s signal with a power $p_{n}^{m}$, and PU $m$ rewards a dedicated access time $t_{n}^{m}$ on its spectrum to SU $n$.

With the AF protocol, the PU $m$ 's transmitter $\mathrm{PT}_{m}$ broadcasts the data (with unit power) in Phase I, and SU $n$ normalizes the received signal $r_{n, m}$ by a factor $\sqrt{p_{n}^{m}} /\left|r_{n, m}\right|$ and forwards it to $\mathrm{PR}_{m}$ in Phase II. The receiver $\mathrm{PR}_{m}$ combines the received signals in Phases I and II with a maximal ratio combining. Essentially, we can view the transmission during Phases I and II as a single-input, two-output complex Gaussian noise channel. Accordingly, the transmission rate during Phases I and II achieved from i.i.d. complex Gaussian inputs is given by the Shannon-Hartley theorem [23]:

$$
R_{n}^{m}=\frac{1}{2} \cdot \log \left(1+\kappa_{d}^{m}+\kappa_{n}^{m}\right),
$$

where $\kappa_{d}^{m}$ is the signal-to-noise ratio (SNR) on the PU $m$ 's direct channel $\left(\mathrm{PT}_{m}\right.$ to $\left.\mathrm{PR}_{m}\right)$, and $\kappa_{n}^{m}$ is the SNR on the relay channel of SU $n$. For convenience, we normalize the PU's direct transmission power into 1 . Then, we have $\kappa_{d}^{m}=\frac{G_{m}^{2}}{\sigma^{2}}$, where $G_{m}$ is the channel gain of the PU $m^{\prime} \mathrm{s}$ direct channel and $\sigma^{2}$ is the noise power, and

$$
\kappa_{n}^{m}=\frac{p_{n}^{m} G_{m, n}^{2} G_{n, m}^{2}}{p_{n}^{m} G_{n, m}^{2}+G_{m, n}^{2}+\sigma^{2}} \cdot \frac{1}{\sigma^{2}},
$$

where $G_{m, n}$ is the channel gain of the channel between $\mathrm{PT}_{m}$ and $\mathrm{ST}_{n}$ (called the 1st relay channel), and $G_{n, m}$ is the channel gain of the channel between $\mathrm{ST}_{n}$ and $\mathrm{PR}_{m}$ (called the 2nd relay channel). Obviously, the SNR $\kappa_{n}^{m}$ increases with the SU's relay power $p_{n}^{m}$.

Since the cooperative period occupies $\frac{T_{m}}{T_{m}+t_{n}^{m}}$ fraction of the total frame, PU $m$ 's effective transmission rate during the entire frame (when cooperating with SU $n$ ) is

$$
\widetilde{R}_{n}^{m}=\frac{T_{m}}{T_{m}+t_{n}^{m}} \cdot \frac{1}{2} \cdot \log \left(1+\kappa_{d}^{m}+\kappa_{n}^{m}\right) .
$$

We define PU $m$ 's utility as the increase of its transmission rate when cooperating with an $\mathrm{SU} n$, i.e.,

$$
\Pi_{n}^{m} \triangleq \widetilde{R}_{n}^{m}-R_{d}^{m}=\frac{T_{m} \cdot \log \left(1+\kappa_{d}^{m}+\kappa_{n}^{m}\right)}{2 \cdot\left(T_{m}+t_{n}^{m}\right)}-R_{d}^{m},
$$

where $R_{d}^{m}=\log \left(1+\kappa_{d}^{m}\right)=\log \left(1+G_{m}^{2} / \sigma^{2}\right)$ is PU $m^{\prime} \mathrm{s}$ direct transmission rate without any relay.

The SU $n$ 's total energy consumption during the entire frame (when cooperating with PU $m$ ) is

$$
S_{n(m)} \triangleq \frac{T_{m}}{2} \cdot p_{n}^{m}+t_{n}^{m} \cdot 1,
$$

where the first term is due to relaying PU $m$ 's traffic, and the second term is due to SU n's own traffic (with a normalized unit transmission power).

We define the SU $n$ 's utility (when cooperating with PU $m$ ) as the difference between the achieved transmission rate and the incurred power cost, denoted by

$$
\Delta_{n}^{m} \triangleq \widetilde{R}_{n(m)}-\frac{C_{n} S_{n(m)}}{T_{m}+t_{n}^{m}}=\frac{t_{n}^{m}\left(R_{n(m)}-C_{n}\right)-p_{n}^{m} \frac{C_{n} T_{m}}{2}}{T_{m}+t_{n}^{m}},
$$

where $\frac{S_{n(m)}}{T_{m}+t_{n}^{m}}$ is the average transmission power, and $C_{n}$ is its sensitivity for unit power consumption.

\section{TWO-Sided MARKet Formulation}

Before the detailed modeling of the spectrum sharing market, we first provide the definition of a basic twosided matching market (also called two-sided market).

Definition 1 (Two-sided Matching Market): A two-sided matching market is a market consisting of two disjoint sets of users, where an user on one side can be matched with only one user on the other side.

A one-to-one (binary) matching (or just matching) in a two-sided market is defined as follows.

Definition 2 (Matching): A one-to-one matching between two disjoint sets $\mathcal{M}$ and $\mathcal{N}$ can be represented by a one-to-one correspondence $\mu(\cdot)$, where $m \in \mathcal{M}$ is mapped to $n \in \mathcal{N}$ (i.e., $\mu(m)=n$ ) if and only if $n$ is also mapped to $m$ (i.e., $\mu(n)=m$ ).

For notational convenience, we will also write $\mu(m)$ as $\mu_{m}$ and $\mu(n)$ as $\mu_{n}$ when there is no confusion caused.

\subsection{Two-sided Market Modeling}

In our model, each PU has different preferences over SUs depending on the locations of SUs, and wants to select the most efficient $\mathrm{SU}$ as relay; each $\mathrm{SU}$ also has different preferences over PUs depending on its channel gains on PUs' bands, and want to obtain the dedicated spectrum access time from the most "generous" PU. Thus, we can 


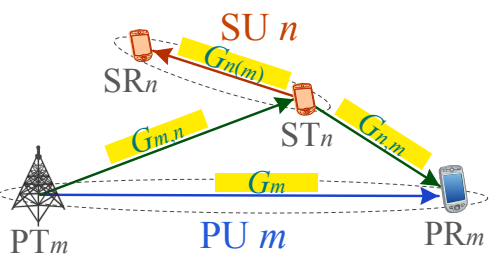

Fig. 3. Illustration of the key network information.

formulate this model as a two-sided market, with PUs on one side and SUs on the other side. A PU is matched to an SU (or equivalently, an SU is matched to a PU) means that the PU cooperates with the SU, that is, the SU relays traffic for the PU, and the PU rewards dedicated spectrum access time to the SU. Clearly, in this twosided market, we need to consider not only the binary matching between PUs and SUs (given in Definition 2), but also the detailed resource exchange between each pair of matched PU and SU ${ }^{5}$

More specifically, for each pair of matched PU and SU, the PU's and SU's utilities depend not only on the SU's relay power (in Phase II), but also on the PU's spectrum access time reward (in Phase III). In addition, an increase in one user's utility will lead to a (not necessarily the same amount of) decrease in the other's utility in the matching pair. Therefore, we face a two-sided market with transferrable utility, where each user's utility (or preference) in a given matching pair is not fixed, but depends on the resource exchange with the matched user (i.e., the access time reward and relay power).

Based on the above discussion, we address the following matching problem for the proposed market:

Question 1 (Relay-Assignment): What kind of matching pairs will emerge from users' strategic interactions?

Question 2 (Resource-Exchange): What is the detailed resource exchange (i.e., the access time and relay power) for each pair of matched PU and SU?

\subsection{Market Equilibrium - Stable Matching}

Now we consider the above matching problem from a game-theoretical analysis. Note that game theory is applicable to our model, as PUs and SUs are rational and self-interested, and always want to maximize their own utilities. Furthermore, one user's decision will affect others' utilities and decisions. To solve the matching problem, it would be useful to understand what kind of matching will be "broken" (and thus unstable).

Definition 3 (Unstable Matching): A matching will be broken if one of the following conditions is satisfied:

(a) There exist a PU and an SU who are not matched to each other but both prefer to be;

(b) There exists a PU or an SU with a negative utility in the existing matching.

5. Since each binary matching is associated with a set of resource exchanges (each for a pair of matched users), we will use the terminology "matching" to denote both (i) the binary matching between PUs and SUs, and (ii) the resource exchange for each pair of matched users.

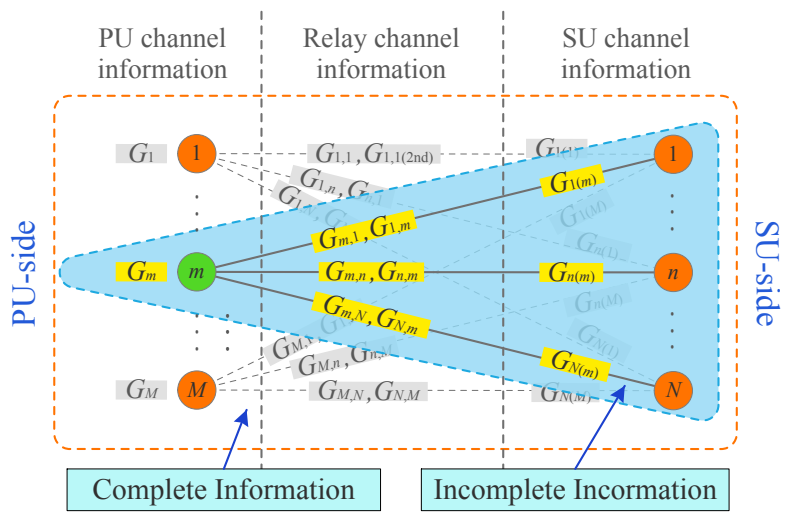

Fig. 4. Illustration of complete and incomplete information scenarios. Under complete information, each PU $m$ knows all network information (in the rectangle). Under incomplete information, each PU $m$ knows its local information (in the blue triangle).

In (a), the PU and the SU would discard their current partners and match to each other. In (b), the PU or SU would remain single rather than stick to the current partner. Thus, a broken matching is "unstable".

As long as a matching is not broken, we call it a stable matching, or equivalently, a market equilibrium.

Definition 4 (Market Equilibrium): A market equilibrium is a stable matching, where no user can improve its utility via unilateral deviation (i.e., choosing another partner or changing resource exchange details).

Based on the above definitions, we can rewrite the matching problem (given in Questions 1 and 2) into the following equivalent question: what market equilibrium (stable matching) will emerge in the two-sided matching market? Notice that there are many important factors affecting the market equilibrium realization, one of which is: which side of the market has the market power to propose cooperation offers to the other side [15]. In this paper, we consider a PU-proposal market, where PUs have the authority to propose offers ${ }^{6}$ That is, each PU decides which SU to select and what time reward and relay power request to/from that $\mathrm{SU}$, and each SU passively accepts or rejects the received offer. The study of a PU-proposal market is motivated by the fact that PUs usually have more market power than SUs in cooperative spectrum sharing, since their number is limited and they own the scarce spectrum licenses.

\subsection{Network Information}

In addition to who are the proposers, the information scenario also affects the equilibrium. That is, which equilibrium will eventually appear also depends on how much network information are known to PUs. In our

6. Note that the analysis for the PU-proposal market can be directly extended to the SU-proposal market, where SUs propose offers to PUs, and PUs simply accept or reject the received offers. 
TABLE 2

Information Scenarios and Associated Market Equilibria.

\begin{tabular}{|c|c|c|c|c|}
\hline Information Scenario & Each PU $m^{\prime}$ s Knowledge & Equilibrium & Property & Section No. \\
\hline \hline Complete & $\left\{G_{m}, G_{n(m)}, G_{m, n}, G_{n, m}\right\}_{n \in \mathcal{N}, m \in \mathcal{M}}$ & PU-Optimal-EQ & Pareto-optimal for PUs & Section 6 \\
\hline \hline Incomplete & $\left\{G_{m}, G_{n(m)}, G_{m, n}, G_{n, m}\right\}_{n \in \mathcal{N}}$ & PU-Robust-EQ & Guaranteed for PUs & Section 7 \\
\hline
\end{tabular}

model, the key network information mainly include ${ }^{7}$

(a) $G_{m}$ : the direct channel gain of each PU $m$;

(b) $G_{n(m)}$ : the direct channel gain of each SU $n$ on each PU $m$ 's spectrum band;

(c) $G_{m, n}$ and $G_{n, m}$ : the relay channel gains between each pair of PU $m$ and SU $n$.

For convenience, we illustrate the network information (between PU $m$ and SU $n$ ) in Figure 3, where the label in each link denotes the associated channel gain.

In this work, we consider two different information scenarios: complete information and incomplete information, depending on how much network information the PUs know. Specifically, in the complete information scenario, each PU $m$ is assumed to know the whole network information, i.e., $\left\{G_{m}, G_{n(m)}, G_{m, n}, G_{n, m}\right\}_{n \in \mathcal{N}, m \in \mathcal{M}}$. In the incomplete information scenario, each PU $m$ is assumed to know its local network information only, i.e., $\left\{G_{m}, G_{n(m)}, G_{m, n}, G_{n, m}\right\}_{n \in \mathcal{N}}$, but not those of other PUs. Note that the incomplete information scenario is more practical, as a PU can use reference signal strength (RSS) to detect the channel gains to all potential SUs, yet it is hard for him to estimate other PUs' channel gains. For convenience, we summarize these information scenarios in Figure $4^{8}$

In what follows, we will characterize the sufficient and necessary conditions for the market equilibrium (of the PU-proposal market) in Section 6, and study which equilibria will emerge in complete and incomplete information scenarios in Section 7 For convenience, we summarize these information scenarios and the associated market equilibria in Table 2 .

\section{A Simplified Model}

Before studying the market equilibrium in the general model, we first consider a simplified model where the access time reward offered by each PU (in Phase III) and the relay power contributed by each SU (in Phase II) are fixed. Thus, we only need to consider the binary matching between PUs and SUs (i.e., the relay assignment problem defined in Question 1. 9

Note that we use this simple model to illustrate some key properties of the proposed matching market, which

7. Notice that we view the cooperative transmission time $T_{m}$ of each PU $m$ and the power sensitivity $C_{n}$ of each SU $n$ as public information. This is because these parameters are usually pre-defined and keep unchanged in a long time, given the types of PUs or SUs.

8. We further study a strongly incomplete information scenario in the online technical report [29], where each PU $m$ does not even know each SU $n$ 's channel gain, i.e., $G_{n(m)}$. The equilibrium analysis for such a stronger incomplete information scenario is similar.

9. This model is similar to the two-sided market model in [15], [20], and our results in this section also refer to those in [15], [20]. is essential for understanding the general model in Sections 6 and 7 Moreover, this simplified model is useful for the network scenario with inflexible relay protocols or static physical layer specifications, where secondary access time and relay power cannot be freely adjusted.

\subsection{Users' Preferences}

With fixed time reward and relay power, each user's utility for each particular partner on the other side is fixed. Thus, the model degenerates to a two-sided matching problem without utility transferring (similar to the Marriage model considered in [15]).

Let $t_{m}$ denote the (fixed) spectrum access time rewarded by PU $m$ for any SU collaborating with him, and $p_{n}$ the (fixed) relaying power offered by SU $n$ for any PU collaborating with him. The preferences of a PU $m$ can be represented by an ordered list in the decreasing preference, $P(m)$, on the SUs, ${ }^{10}$

$$
P(m)=\left\{n_{1}, n_{2}, \ldots, n_{p}\right\},
$$

subject to (i) $\Pi_{n_{i}}^{m}\left(t_{m}, p_{n_{i}}\right) \geq \Pi_{n_{j}}^{m}\left(t_{m}, p_{n_{j}}\right)$ if $i<j$, and (ii) $\Pi_{n}^{m}\left(t_{m}, p_{n}\right) \geq 0$ for any SU $n \in P(m)$, and $\Pi_{n^{\prime}}^{m}\left(t_{m}, p_{n^{\prime}}\right)<0$ for any SU $n^{\prime} \notin P(m)$. Here $\Pi_{n}^{m}(\cdot)$ is the PU $m^{\prime}$ 's utility defined in (4). The first condition means that PU $m$ prefers an SU who provides him a higher utility, and the second condition means that PU $m$ never selects an SU who brings him a negative utility.

Similarly, the preference list of an SU $n$ on the PUs can be represented by

$$
Q(n)=\left\{m_{1}, m_{2}, \ldots, m_{q}\right\},
$$

subject to (i) $\Delta_{n}^{m_{i}}\left(t_{m_{i}}, p_{n}\right) \geq \Delta_{n}^{m_{j}}\left(t_{m_{j}}, p_{n}\right)$ if $i<j$, and (ii) $\Delta_{n}^{m}\left(t_{m}, p_{n}\right) \geq 0$ if $m \in Q(n)$, and $\Delta_{n}^{m^{\prime}}\left(t_{m^{\prime}}, p_{n}\right)<0$ $m^{\prime} \notin Q(n)$. Here $\Delta_{n}^{m}(\cdot)$ is the SU $n^{\prime}$ s utility in 8 .

\subsection{An Example and Its Equilibrium}

We provide an example to illustrate the equilibria under the fixed preferences ${ }^{11}$ Recall that a binary matching between PUs and SUs is represented by a correspondence $\mu(\cdot)$, where $\mu_{m}=n$ (or equivalently, $\mu_{n}=m$ ) means PU $m$ is matched to SU $n$, and $\mu_{m}=\emptyset$ or $\mu_{n}=\emptyset$ means PU $m$ or SU $n$ remains single (i.e., not engaged in the cooperative spectrum sharing).

Example 1: Consider a two-sided market with 3 PUs $\left(m_{1}, m_{2}, m_{3}\right)$ and 3 SUs $\left(n_{1}, n_{2}, n_{3}\right)$ with preferences:

10. The number of elements in set $P(m)$ could be smaller than $N$, that is, PU $m$ is only interested in a subset of SUs.

11. The complete equilibrium analysis for such a simplified model (with the fixed preferences) can be referred to [29]. 


\begin{tabular}{c|c||c|c}
\hline \multicolumn{2}{c||}{ PUs' preferences } & \multicolumn{3}{c}{ SUs' preferences } \\
\hline$P\left(m_{1}\right)$ & $n_{1}, n_{2}, n_{3}$ & $Q\left(n_{1}\right)$ & $m_{2}, m_{3}, m_{1}$ \\
\hline$P\left(m_{2}\right)$ & $n_{2}, n_{3}, n_{1}$ & $Q\left(n_{2}\right)$ & $m_{3}, m_{1}, m_{2}$ \\
\hline$P\left(m_{3}\right)$ & $n_{3}, n_{1}, n_{2}$ & $Q\left(n_{3}\right)$ & $m_{1}, m_{2}, m_{3}$ \\
\hline
\end{tabular}

According to the definition of equilibrium in Definition 4. we can easily obtain the following three equilibria $\mu^{a}, \mu^{b}$, and $\mu^{c}$ for the above example:

\begin{tabular}{c|c|c|c||c|c|c||c|c|c}
\hline Equilibria & \multicolumn{3}{|c|}{$\mu^{a}(\cdot)$} & \multicolumn{3}{c||}{$\mu^{b}(\cdot)$} & \multicolumn{3}{c}{$\mu^{c}(\cdot)$} \\
\hline PUs & $m_{1}$ & $m_{2}$ & $m_{3}$ & $m_{1}$ & $m_{2}$ & $m_{3}$ & $m_{1}$ & $m_{2}$ & $m_{3}$ \\
\hline SUs & $n_{1}$ & $n_{2}$ & $n_{3}$ & $n_{2}$ & $n_{3}$ & $n_{1}$ & $n_{3}$ & $n_{1}$ & $n_{2}$ \\
\hline
\end{tabular}

Let us check the feasibility of the above equilibria using the definition of market equilibrium. Take the equilibrium $\mu^{b}$ as an example, where PU $m_{1}$ is matched to $\mathrm{SU} n_{2}, \mathrm{PU} m_{2}$ is matched to SU $n_{3}$, and PU $m_{3}$ is matched to SU $n_{1}$. We can easily find that PU $m_{1}$ prefers SU $n_{1}$ than its current partner SU $n_{2}\left(\right.$ as $\left.\Pi_{n_{2}}^{m_{1}}<\Pi_{n_{1}}^{m_{1}}\right)$, but SU $n_{1}$ has no incentive to discard its current partner PU $m_{3}$ and pair with PU $m_{1}$ (as $\Delta_{n_{1}}^{m_{3}}>\Delta_{n_{1}}^{m_{1}}$ ). Similarly, PU $m_{2}$ prefers SU $n_{2}$ than its current partner SU $n_{3}$, but SU $n_{2}$ has no incentive to discard its current partner PU $m_{1}$ and pair with PU $m_{2}$. PU $m_{3}$ prefers SU $n_{3}$ than its current partner SU $n_{1}$, but SU $n_{1}$ has no incentive to discard its current partner PU $m_{2}$ and pair with PU $m_{3}$. Thus, there does not exist a pair of PU and SU who are not matched to each other but both prefer to be.

\section{Market Equilibrium Analysis}

Now we characterize the market equilibrium in a general model where the access time reward and the relay power are not fixed. In this case, each user's utility (with a particular partner at the other side) is no longer fixed, but changes with the resource exchange (i.e., the access time reward and relay power) between them. Accordingly, the preference list of each user depends on its current resource exchanges with users at the other side. Thus, we need to consider not only the binary matching between PUs and SUs (i.e., the relay assignment problem defined in Question 1), but also the resource exchange between each pair of matched users (i.e., the resource exchange problem defined Question 2).

In what follows, we will start by defining the utility transfer function, which captures the interaction within each matching pair. Then we characterize the necessary and sufficient conditions for market equilibrium. We will show that there are multiple (infinite number) of market equilibria. In the next section, we will further study which specific equilibrium will actually emerge in different information scenarios.

\subsection{Utility Transfer Function - UTF}

Suppose that PU $m$ is matched to SU $n$. We first study how their utilities change with the access time reward and the relay power.

Recall in the PU-proposal market, PUs propose the offers and SUs decide whether to accept or not. Let $\delta_{n}^{m}$ denote the SU $n$ 's highest utility from all other PUs'
TABLE 3

Important Notations in Section 6

\begin{tabular}{ll}
\hline$f_{n}^{m}(\cdot)$ & PU $m^{\prime}$ 's maximal utility given the matched SU's utility; \\
$g_{n}^{m}(\cdot)$ & SU $n$ 's maximal utility given the matched PU's utility; \\
\hline$\mu_{n}$ & The PU matched to SU $n ;$ \\
$\mu_{m}$ & The SU matched to PU $m ;$ \\
$\delta_{n}$ & The SU $n$ 's achieved utility in a given matching; \\
$\bar{\delta}_{n}$ & The SU $n$ 's highest achievable utility in a matching; \\
$\underline{\delta}_{n}$ & The SU $n$ 's lowest acceptable utility in a matching; \\
\hline
\end{tabular}

offers (except PU $m^{\prime} \mathrm{s}$ ) and from remaining single (not matched to any PU), called reservation utility of SU $n$ (towards PU $m)^{12}$ Then, to attract SU $n$ 's cooperation, PU $m$ has to offer SU $n$ a utility no less than $\delta_{n}^{m}$. Thus, the PU $m$ 's maximum utility is given by

$$
\begin{aligned}
\Pi_{n}^{m *}= & \max _{\left\{p_{n}^{m}, t_{n}^{m}\right\}} \Pi_{n}^{m}\left(p_{n}^{m}, t_{n}^{m}\right) \\
& \text { s.t. } \quad \Delta_{n}^{m}\left(p_{n}^{m}, t_{n}^{m}\right) \geq \delta_{n}^{m},
\end{aligned}
$$

where $\Pi_{n}^{m}\left(p_{n}^{m}, t_{n}^{m}\right)$ is PU $m^{\prime}$ s utility defined in (4), $\Delta_{n}^{m}\left(p_{n}^{m}, t_{n}^{m}\right)$ is SU $n$ 's utility defined in (8). Notice that the constraint must be tight at the optimality. This implies that the variable $t_{n}^{m}$ (or $p_{n}^{m}$ ) can be rewritten as a function of $p_{n}^{m}$ (or $t_{n}^{m}$ ), and thus the problem (11) can be transformed into a single-variable optimization problem. Hence, problem (11) can be solved efficiently using many one-dimension exhaustive search methods. We denote the computational complexity of solving (11) as $\rho$.

It is easy to check that the PU m's maximal utility $\Pi_{n}^{m *}$ given by (11) is strictly decreasing in SU $n$ 's reservation utility $\delta_{n}^{m}$. Thus, we can write $\Pi_{n}^{m *}$ as a decreasing function of $\delta_{n}^{m}$ :

$$
\Pi_{n}^{m *} \triangleq f_{n}^{m}\left(\delta_{n}^{m}\right)
$$

We refer to $f_{n}^{m}(\cdot)$ as the Utility Transfer Function (UTF). Furthermore, we denote

$$
g_{n}^{m}(\cdot) \triangleq f_{n}^{m(-1)}(\cdot)
$$

as the Inverse Utility Transfer Function (IUTF), i.e., $\pi_{n}^{m}=f_{n}^{m}\left(\delta_{n}^{m}\right)$ if and only if $\delta_{n}^{m}=g_{n}^{m}\left(\pi_{n}^{m}\right)$. Note that $f_{n}^{m}\left(\delta_{n}^{m}\right)$ denotes the PU $m^{\prime}$ 's maximal utility when giving SU $n$ a utility $\delta_{n}^{m}$, and $g_{n}^{m}\left(\pi_{n}^{m}\right)$ denotes the SU $n^{\prime}$ ' maximal utility when giving PU $m$ a utility $\pi_{n}^{m}$

To facilitate the understanding of the later analysis, we list some important notations in Table 3.

\subsection{Sufficient \& Necessary Conditions}

Now we study the sufficient and necessary conditions for an equilibrium. The optimization problem (11) shows that the PU $m$ 's maximal utility depends on $S U n^{\prime}$ s utility $\delta_{n}^{m}$, so does the optimal access time $t_{n}^{m}$ and relay power $p_{n}^{m}$. Thus, the resource exchange problem in Question 2 is equivalent to the following utility division problem:

12. Note that an $\mathrm{SU} n$ 's reservation utility is PU-dependent, i.e., $\delta_{n}^{m_{1}}$ may be different from $\delta_{n}^{m_{2}}$, as it is the SU's highest utility from all but one of the PUs.

13. An illustration of UTF is provided by Figure 6 in [29]. 
what is the utility for each SU in a matching? This means that we can rewrite a matching as

$$
\left\{\left(\mu_{n}, \delta_{n}\right), \forall n \in \mathcal{N}\right\}
$$

where $\mu_{n}$ denotes the PU matched to SU $n$, and $\delta_{n}$ denotes the SU $n$ 's utility in the given matching ${ }^{14} \mathrm{Ob}$ viously, in such a matching, the utility of PU $\mu_{n}$ (i.e., the PU matched to SU $n)$ is $f_{n}^{\mu_{n}}\left(\delta_{n}\right)$, which is PU $\mu_{n}$ 's maximum achievable utility computed by (11). Note that the utility is zero for an unmatched SU $n$ (with $\mu_{n}=\emptyset$ ) or PU $m$ (with $m \neq \mu_{n}, \forall n \in \mathcal{N}$ ).

In what follows, we will present the lower-bound and upper-bound of each $\delta_{n}$ (for the matched PU $\mu_{n}$ and SU $n$ ), such that none of PUs and SUs has an incentive to break the current matching. To achieve this, for each pair of matched PU $\mu_{n}$ and SU $n$ : (i) PU $\mu_{n}$ (or SU n) has no incentive to break the current matching by remaining single, or by pairing with another SU (or PU), and (ii) all PUs other than $\mu_{n}$ (or all SUs other than $n$ ) have no incentives to discard their respective partners and pair with SU $n$ (or PU $\mu_{n}$ ).

According to Definition 4, we first have the following necessary conditions for an equilibrium.

Lemma 1: If $\left\{\left(\mu_{n}, \delta_{n}\right), \forall n\right\}$ is an equilibrium, then the following conditions hold: for each SU $n \in \mathcal{N}$,

(IR) $f_{n}^{\mu_{n}}\left(\delta_{n}\right) \geq 0$;

(IC) $f_{n}^{\mu_{n}}\left(\delta_{n}\right) \geq f_{k}^{\mu_{n}}\left(\delta_{k}\right), \quad \forall k \neq n$,

where $\mu_{n}$ denotes the PU matched to SU $n$.

The first condition is generally referred to as Individual Rationality (IR), and the second condition is generally referred to as Incentive Compatibility (IC). The proof follows directly from the definition of equilibrium: If the IR condition is violated by a PU $\mu_{n}$, then PU $\mu_{n}$ will discard its partner (SU $n$ ) and remain single; If the IC condition is violated by a PU $\mu_{n}$, i.e., there exists another SU $k \neq n$ such that $f_{n}^{\mu_{n}}\left(\delta_{n}\right)<f_{k}^{\mu_{n}}\left(\delta_{k}\right)$, then PU $\mu_{n}$ can get a better utility by paring up with SU $k$ instead ${ }^{15}$

The IC and IR conditions for a PU $\mu_{n}$ ensure that PU $\mu_{n}$ has no incentive to change its decision. We further introduce the Competitive Compatibility (CC) condition for PU $\mu_{n}$, which ensures that no other PUs has the incentive to compete with a PU $\mu_{n}$ (for SU $n$ ). Intuitively, the CC condition for a PU $\mu_{n}$ (i.e., the PU matched to SU n) means no other PU $m$ can achieve a higher utility on SU $n$ than on its current partner SU $\mu_{m}$. Formally,

Lemma 2: If $\left\{\left(\mu_{n}, \delta_{n}\right), \forall n\right\}$ is an equilibrium, then the following condition holds: for each PU $\mu_{n} \in \mathcal{M}{ }^{16}$

(CC) $f_{\mu_{m}}^{m}\left(\delta_{\mu_{m}}\right) \geq f_{n}^{m}\left(\delta_{n}\right), \quad \forall m \neq \mu_{n}$

where $\mu_{m}$ denotes the SU matched to PU $m$.

The IR condition for a PU $\mu_{n}$ implies

$$
f_{n}^{\mu_{n}}\left(\delta_{n}\right) \geq 0 \Rightarrow \delta_{n} \leq g_{n}^{\mu_{n}}(0),
$$

14. Note we omit the superscript $\mu_{n}$ in $\delta_{n}^{\mu_{n}}$ for simplicity of writing. 15. With a proper offer from PU $\mu_{n}$ (e.g., giving SU $k$ a utility $\delta_{k}+\epsilon$, where $\epsilon$ is any infinitesimal positive number), SU $k$ will also improve its utility through the new pairing and thus will accept.

16 . Note that $f_{\mu_{m}}^{m}(\cdot) \equiv 0$ if $\mu_{m}=\emptyset$, which denotes the utility of PU $m$ when not engaging in any collaboration. where $g_{n}^{\mu_{n}}(0)=f_{n}^{\mu_{n}(-1)}(0)$ is the maximum achievable utility of SU $n$ when leaving zero utility to PU $\mu_{n}$. The IC condition for a PU $\mu_{n}$ implies

$$
\begin{aligned}
& f_{n}^{\mu_{n}}\left(\delta_{n}\right) \geq f_{k}^{\mu_{n}}\left(\delta_{k}\right), \quad \forall k \neq n \\
\Rightarrow & \delta_{n} \leq g_{n}^{\mu_{n}}\left(f_{k}^{\mu_{n}}\left(\delta_{k}\right)\right), \quad \forall k \neq n \\
\Rightarrow & \delta_{n} \leq \min _{k \neq n}\left\{g_{n}^{\mu_{n}}\left(f_{k}^{\mu_{n}}\left(\delta_{k}\right)\right)\right\},
\end{aligned}
$$

where $g_{n}^{m}(x)=f_{n}^{m(-1)}(x)$ is the SU $n^{\prime}$ s maximum achievable utility when leaving a utility $x$ to PU $m$. The CC condition for a PU $\mu_{n}$ further implies

$$
\begin{aligned}
& \delta_{n} \geq g_{n}^{m}\left(f_{\mu_{m}}^{m}\left(\delta_{\mu_{m}}\right)\right), \quad \forall m \neq \mu_{n} \\
\Rightarrow & \delta_{n} \geq \max _{m \neq \mu_{n}}\left\{g_{n}^{m}\left(f_{\mu_{m}}^{m}\left(\delta_{\mu_{m}}\right)\right)\right\} .
\end{aligned}
$$

Based on the above discussions, we can obtain the lowest acceptable utility $\underline{\delta}_{n}$ and the highest achievable utility $\bar{\delta}_{n}$ for an SU $n$ in an equilibrium $\left\{\left(\mu(n), \delta_{n}\right), \forall n\right\}$, i.e.,

$$
\begin{aligned}
& \underline{\delta}_{n} \triangleq \max _{m \neq \mu_{n}}\left\{g_{n}^{m}\left(f_{\mu_{m}}^{m}\left(\delta_{\mu_{m}}\right)\right), 0\right\}, \\
& \bar{\delta}_{n} \triangleq \min _{k \neq n}\left\{g_{n}^{\mu_{n}}\left(f_{k}^{\mu_{n}}\left(\delta_{k}\right)\right), g_{n}^{\mu_{n}}(0)\right\} .
\end{aligned}
$$

Intuitively, PU $\mu_{n}$ cannot offer a utility $\delta_{n}$ lower than $\underline{\delta}_{n}$ to SU $n$, otherwise some other PU $m \neq \mu_{n}$ will have the incentive to pair up with SU $n$ (by offering SU $n$ a utility slightly higher than $\underline{\delta}_{n}$ ). This will make the original matching broken. On the other hand, PU $\mu_{n}$ will never offer a utility $\delta_{n}$ higher than $\bar{\delta}_{n}$ to SU $n$, otherwise PU $\mu_{n}$ would be better off by pairing up with an other SU. This will also make the matching broken.

Therefore, we can rewrite the necessary conditions in Lemma 1 more explicitly as follows: If a matching $\left\{\left(\mu_{n}, \delta_{n}\right), \forall n\right\}$ is an equilibrium, the condition holds:

$$
\underline{\delta}_{n} \leq \delta_{n} \leq \bar{\delta}_{n}, \quad \forall n \in \mathcal{N},
$$

where $\underline{\delta}_{n}$ and $\bar{\delta}_{n}$ are defined in (13) and (14), respectively. The left inequality is due to the CC condition, and the right inequality is due to the IC and IR conditions.

The next theorem shows the above conditions are not only necessary, but also sufficient.

Theorem 1 (Sufficient \& Necessary Condition): A matching $\left\{\left(\mu_{n}, \delta_{n}\right), \forall n\right\}$ is an equilibrium, if and only if

$$
\underline{\delta}_{n} \leq \delta_{n} \leq \bar{\delta}_{n}, \quad \forall n \in \mathcal{N} .
$$

Due to space limit, we put the detailed proof in [29].

\subsection{Property of Equilibrium}

To facilitate the characterization of equilibrium in different information scenarios (in the next section), we first show the following property for equilibrium.

Lemma 3 (Generalized Lattice Theorem): Given any possible binary matching $\mu(\cdot)$, if $\left\{\left(\mu_{n}, \delta_{n}^{I}\right), \forall n\right\}$ and $\left\{\left(\mu_{n}\right.\right.$, $\left.\left.\delta_{n}^{I I}\right), \forall n\right\}$ are equilibria, then the matching $\left\{\left(\mu_{n}, \delta_{n}^{X}\right), \forall n\right\}$ with $\delta_{n}^{X}=\min \left(\delta_{n}^{I}, \delta_{n}^{I I}\right)$ is also an equilibrium.

Lemma 3 can be seen as a generalization of the Lattice Theorem proposed by Kunth [24] for the basic two-sided matching (without utility transferring). By repeatedly 
applying Lemma 3 on any two equilibria, we will reach the unique optimal equilibrium for PUs.

Theorem 2 (Optimality): There exists a Pareto-optimal equilibrium $\left\{\left(\mu_{n}, \delta_{n}^{*}\right), \forall n\right\}$ for PUs, where every PU achieves its maximum utility among all equilibria. In addition, $\left\{\left(\mu_{n}, \delta_{n}^{*}\right), \forall n\right\}$ satisfies ${ }^{17}$

$$
\delta_{n}^{*}=\underline{\delta}_{n}^{*}, \quad \forall n \in \mathcal{N} .
$$

Theorem 2 not only shows the existence of the Paretooptimal equilibrium for PUs, but also suggests a method to calculate the Pareto-optimal equilibrium for PUs. Specifically, given any feasible matching $\mu(\cdot)$, we can derive each SU's utility (and the associated time reward and relay power) under the Pareto-optimal equilibrium by jointly solving the following function set:

$$
\delta_{n}=\max _{m \neq \mu_{n}}\left\{g_{n}^{m}\left(f_{\mu_{m}}^{m}\left(\delta_{\mu_{m}}\right)\right), 0\right\}, \forall n \in \mathcal{N} .
$$

Obviously, if there is a unique solution for (15), it must be the unique Pareto-optimal equilibrium. If there is no solution for (15), the matching $\mu(\cdot)$ is not feasible.

However, directly solving the above function set is difficult. Consider a single equation in the function set (say, $\left.\delta_{n}\right)$. To solve this equation, we first need to compute the utilities of all PUs other than $\mu_{n}$ on their own matching pairs, i.e., $f_{\mu_{m}}^{m}\left(\delta_{\mu_{m}}\right), \forall m \neq \mu_{n}$. Then, we need to compute the SU $n$ 's maximum achievable utility when giving each PU $m \neq \mu_{n}$ a utility $f_{\mu_{m}}^{m}\left(\delta_{\mu_{m}}\right)$, i.e., $g_{n}^{m}\left(f_{\mu_{m}}^{m}\left(\delta_{\mu_{m}}\right)\right)$, $\forall m \neq \mu_{n}$. Finally, the SU $n$ 's utility $\delta_{n}$ is the maximum of the above computed maximum achievable utilities and zero. Obviously, for this single equation, we need to repeatedly solve the optimization problem (11) $2 \cdot(M-1)$ times. Furthermore, jointly solving $N$ equations makes the problem even more complicated. This motivates us to study low complexity algorithms to realize different market equilibria in the next section.

\section{Market Equilibrium Realization}

In the previous section, we characterized the necessary and sufficient conditions of market equilibrium. In this section, we will study which equilibrium will actually emerge in different information scenarios.

Specifically, we consider two typical information scenarios: complete and incomplete information, depending on how much network information the PUs know (see Section 4.3 for details). Notice that in the PU-proposal market, PUs propose offers and SUs respond by rejecting or accepting offers. Under complete information, each PU knows the whole network information, and can precisely predict the SUs' preferences. Thus, SUs cannot mis-represent their preferences, and have to respond in the truthful manner. That is, each SU will always accept the best offer that brings him the maximum positive utility. Under incomplete information, however, each PU

17. Similarly, there always exists a Pareto-optimal equilibrium $\left\{\left(\mu_{n}, \delta_{n}^{*}\right), \forall n\right\}$ for SUs, where every SU achieves its maximum utility among all equilibria. In addition, $\left\{\left(\mu_{n}, \delta_{n}^{*}\right), \forall n\right\}$ satisfies: $\delta_{n}^{*}=\bar{\delta}_{n}^{*}, \forall n$. knows only its local network information, and cannot predict the SUs' preferences with other PUs. Thus, SUs can potentially mis-represent their preferences, and respond in a non-truthful manner. That is, each SU may reject the best offer that brings him the maximum positive utility, as long as such a mis-representation can bring him a better utility.

\subsection{Equilibrium under Complete Information}

Under complete information, each SU has to respond in a truthful manner, and accept the best offer that brings him the maximum positive utility. In this sense, we can view the SUs' (truthful) responses as natural reactions. Therefore, the matching problem can be viewed as an $M$-player game with complete information, where game players are PUs, whose objectives are to maximize their own utilities. By (11), each PU's maximal utility strictly decreases with the utility of the matched SU. Thus, given all other PUs' offers (strategies), the best strategy for a particular PU $\mu_{n}$ (i.e., the PU matched to $\mathrm{SU} n$ ) is to offer the lowest acceptable utility to its matched SU n, i.e., $\delta_{n}=\underline{\delta}_{n}$. This essentially lead to the Pareto-optimal equilibrium for PUs (PU-Optimal-EQ).

Next we show how to compute the PU-Optimal-EQ in the complete information scenario. Notice that when $\mu(\cdot)$ is given, the PU-Optimal-EQ can be computed by jointly solving (15), which is complicated as mentioned previously. Moreover, an exhaustive search of all feasible $\mu(\cdot)$ requires going through a total of $M$ ! possibilities, which has an exponential-time complexity. This makes the solving process mathematically intractable.

To this end, we introduce a "Generalized Deferred Acceptance" (G-DAC) algorithm in Algorithm 1, which converges to the PU-Optimal-EQ in polynomial-time complexity. Specifically, in the G-DAC algorithm, each PU $m$ maintains a vector $\Delta^{m}=\left(\delta_{1}^{m}, \ldots, \delta_{N}^{m}\right)$, which records the utilities it is willing to offer to each SU. The basic idea of the G-DAC algorithm is: (i) in each round, each PU $m$ proposes to the best $\mathrm{SU}$ based on $\Delta^{m}$, and each $\mathrm{SU}$ $n$ accepts the best proposal and rejects others; and (ii) if PU $m$ is rejected by SU $n$ in a round, it increases the offer $\delta_{n}$ by a small positive value $\epsilon$ in the following rounds.

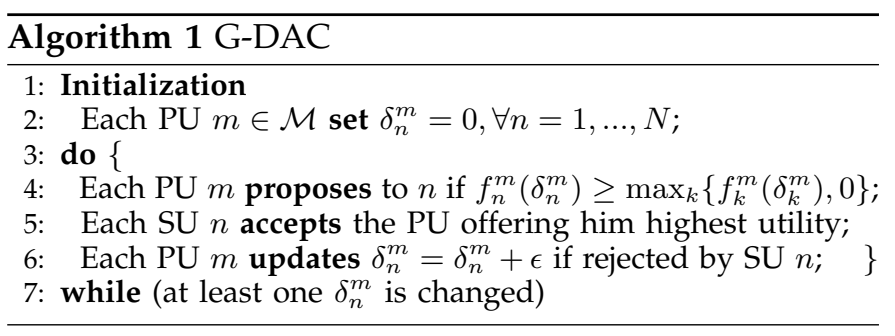

The G-DAC algorithm generalizes the DAP algorithm [15] in the following aspect. In the DAP algorithm, when a PU is rejected by an SU, it will propose to another SU in later rounds (as the SU who has rejected its offer will never accept its offer in the future); while in the G-DAC algorithm, the PU will continue to increase the utility to 
the SU (attempting to attract the SU), until the offer is accepted by the SU or the PU can achieve a higher utility by proposing an offer to another SU ${ }^{18}$ Hence, it brings an additional degree of freedom for PUs making their decisions. Namely, each PU decides not only the target SU that it is going to propose to, but also the utility that it is going to transfer to the SU.

Lemma 4: The G-DAC algorithm converges to the Pareto-optimal equilibrium for PUs (PU-Optimal-EQ), if the stepsize $\epsilon$ is small engouth.

To prove the above lemma, we can first show that the convergence state of G-DAC satisfies the conditions in Theorem 11 and is therefore an equilibrium. Then we can prove the optimality by showing that the G-DAC will stop at the first equilibrium it achieves, which is exactly the Pareto-optimal equilibrium for PUs.

Next we discuss the communication overhead and computational complexity of the G-DAC algorithm. In each round, each PU $m$ needs to send one request message to a particular SU, and each $\mathrm{SU} n$ needs to send back one acceptance message to a particular PU. Thus, the communication overhead is linear in the number of PU-SU pairs (and hence is low). In terms of computation, each PU $m$ needs to solve the optimization problem (11) at most once with complexity $\rho$ in each round (except the first round where the PU needs to solve the optimization problem (11) $N$ times with complexity $N \rho$ ), since at most one price in the vector $\Delta^{m}=\left(\delta_{1}^{m}, \ldots, \delta_{N}^{m}\right)$ is changed in each round. Furthermore, each PU needs to find the maximal utility among $N$ utilities, and each SU needs to find the best offer among at most $M$ proposals. Thus, the total computational complexity is $\mathcal{O}(T \cdot(M \rho+M N+N M))$, where $T=\frac{\sum_{n \in \mathcal{N}} \underline{\underline{\varepsilon}}_{n}^{*}}{\epsilon}$ is the maximum possible iterations, and $\rho$ is the complexity of solving problem (11).

\subsection{Equilibrium under Incomplete Information}

Under incomplete information, each SU can respond in a non-truthful manner, and may reject the best offer that brings him the maximum positive utility, as long as such a mis-representation can bring a better utility for the SU. This can be seen from the following example.

Example 2: Suppose PU $\mu_{n}$ proposes the lowest acceptable utility to its matched SU $n$ (as in the complete information scenario). The SU $n$ can simply reject this offer (which is its best offer for SU $n$ according to the definition of the lowest acceptable utility), such that PU $\mu_{n}$ increases the utility offered to SU $n$.

By the above example, we can see that different misrepresentation behaviors of SUs (e.g., determining when to accept a PU's offer, and when to reject) may lead to different market equilibria. Unfortunately, due to the

18. Intuitively, the G-DAC algorithm is equivalent to an EnglishAuction [25] with SUs as auctioneers and PUs as bidders. The auction works as follows: (i) each SU asks a "price" (the required utility), and gradually increases the price if multiple PUs bid to him, and (ii) each PU submits bid to the best $\mathrm{SU}$, according to the maximum utilities that it can achieve from SUs at the current price level of all SUs. continuity of utility, there are infinite number of misrepresentation behaviors of SUs. Thus, it is impossible to characterize all possible mis-representation behaviors of SUs. To this end, we will focus on characterizing a robust equilibrium for PUs (PU-Robust-EQ), which provides every PU a guaranteed utility under any possible misrepresentations of SUs.

Similarly, the robust equilibrium for PUs (PU-RobustEQ) can be achieved by a "Generalized Reversed Deferred Acceptance" (G-RDAC) algorithm in Algorithm 2, which is same as the G-DAC Algorithm 1 except that it reverses the roles of PUs and SUs in proposing offers ${ }^{19}$ Since the G-DAC algorithm and G-RDAC algorithm are symmetric, we can easily show that the G-RDAC algorithm converges to the Pareto-optimal equilibrium for SUs, which is also the worst-case equilibrium for PUs. Therefore, every PU is guaranteed to achieve a utility no worse than that in this worst-case equilibrium, under any possible misrepresentations of SUs. In this sense, we refer to this worst-case equilibrium for PUs as the robust equilibrium for PUs.

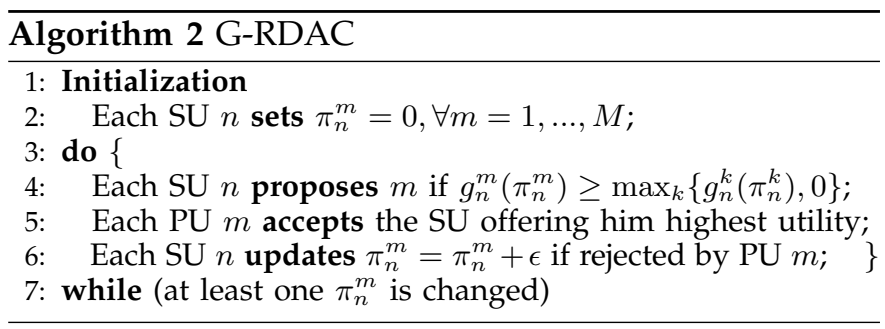

\subsection{Equilibrium under Arbitrary Information}

It is important to note that the PU-Robust-EQ guarantees every PU's lowest achievable utility among all equilibria, while the PU-Optimal-EQ ensures every PU's highest achievable utility among all equilibria. This implies that under any possible equilibrium (in any information scenario), every PU's utility must be bounded by its utilities under the PU-Robust-EQ and the PU-Optimal-EQ. Our numerical studies in the next section further show that in a typical market where the number of PUs and SUs are different, the gap between the PU-Robust-EQ and the PU-Optimal-EQ is quite small. Hence, the PU-Robust$E Q$ or PU-Optimal-EQ can be viewed as an effective approximation to understand all equilibria.

\section{Simulation Results}

To highlight the performance gain achieved from cooperative spectrum sharing, we focus on such a network scenario in simulations, where PUs' direct channel gains are much smaller than SUs' relay channel gains. This may happen when PUs' direct links are highly attenuated due to shadow fading or obstacles. This is very intuitive: only when an SU can provide a large enough benefit to a

19. Similarly, we can show that the G-RDAC algorithm is equivalent to an English-auction with PUs as auctioneers and SUs as bidders. 

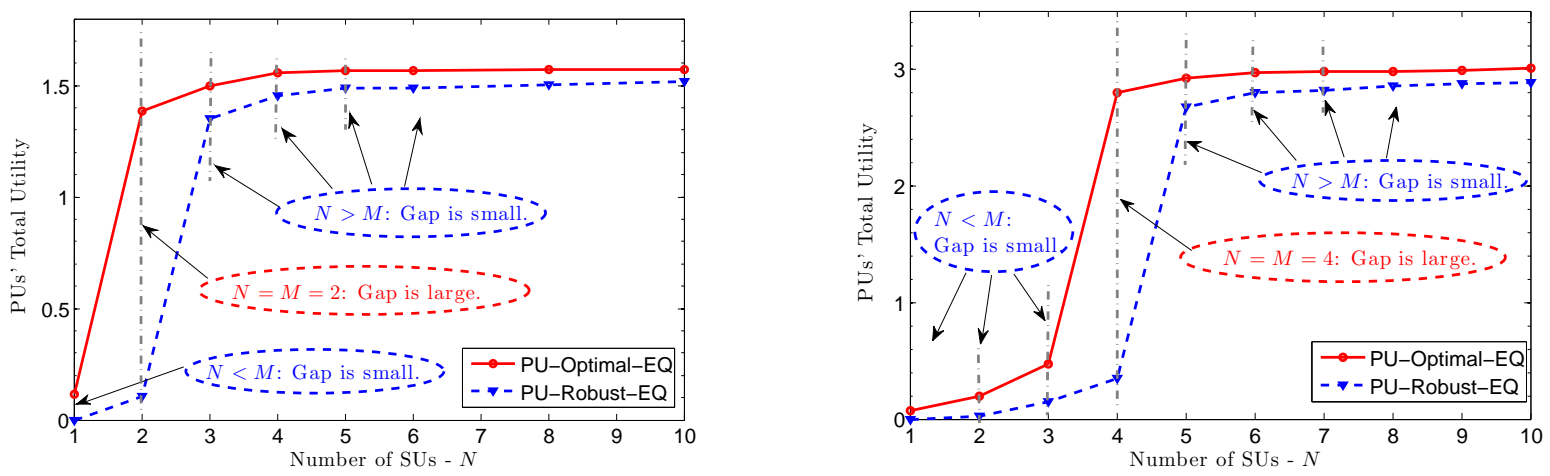

Fig. 5. Total PUs' utility vs Number of SUs $N$. The number of PUs is $M=2$ in the left figure, and $M=4$ in the right figure. The PUs' utility gap between PU-Optimal-EQ and PU-Robust-EQ is less than $10 \%$ when $M \neq N$.

$\mathrm{PU}$, the PU has the incentive to cooperating with the $\mathrm{SU}$, which is a prerequisite of the cooperative spectrum sharing studied in this paper.

We distribute PUs' and SUs' transceiver pairs in a square area of size $1500 \times 1500 \mathrm{~m}^{2}$, and set the distance of each PU's transceiver pair close to $1000 \mathrm{~m}$ and the distance of each SU's transceiver pair close to $400 \mathrm{~m}$ (which are the typical transmission ranges between mobile phones and cellular base stations). Unless otherwise stated, the following default network setting will be used: (i) $C_{n}=T_{m}=1$, (ii) $\sigma^{2}=-105 \mathrm{dBm}$, and (iii) the channel gains are chosen based on the free-space path loss model in [26].

We evaluate the PUs' performance under different market equilibria (illustrations of equilibria can be found in [29]). Note that the SUs' performance can be estimated directly from the conflicting interests between PUs and SUs, that is, an equilibrium that is better for all PUs must be worse for all SUs. In the following, each result is the average of 1000 simulations with randomly generated network topologies.

Figure 5 illusrates the PUs' total utilities vs the number of SUs $N$ under different market equilibria, where the number of PUs is $M=2$ in the left figure, and $M=4$ in the right figure. The red curve denotes the total PUs' utility under the PU-Optimal-EQ, and the blue dash curve denotes the total PUs' utility under the PU-RobustEQ. Obviously, the total PUs' utility under any other equilibrium is bounded by these two curves.

From Figure 5, we can see that the PUs' total utility under equilibrium increases with the number of SUs $N$ (as the competition among SUs increases with $N$ ), and decreases with the number of PUs $M$ (as the competition among PUs increases with $M$ ). Moreover, when $M<N$, PUs can extract most of the generated utility (as the competition among SUs is more intense), and when $M>N$, SUs can extract most of the generated utility (as the competition among PUs is more intense). We further have the following observations.

(1) In the general case where the numbers of PUs and SUs are different (i.e., $M \neq N$ ), the utility gap between PU-Optimal-EQ and PU-Robust-EQ is quite small (e.g., less than $10 \%$ in both figures). This implies that the information scenario (i.e., the amount of network infor- mation that PUs know) has a small impact on the market equilibrium.

(2) In the special case where the numbers of PUs and SUs are identical (i.e., $M=N$ ), the utility gap between PU-Optimal-EQ and PU-Robust-EQ is large. This implies that the information scenario has a significant impact on the market equilibrium.

Intuitively, when $N \neq M$, the imbalance of competition among PUs and among SUs reduces the effect of information incompleteness. The reason is as follows. If the number of SUs is larger than that of PUs $(N>M)$, the intensified competition among SUs pushes SUs to reveal their preferences close to the true values. If the number of PUs is larger than that of SUs $(M>N)$, the intensified competition among PUs already drives most of the generated welfare to the SU-side even in the worstcase equilibrium for SUs, and thus the SUs' performance gain in other equilibria is small. When $M=N$, however, none of the two sides has a dominated competition level in the market, thus the effect of information incompleteness becomes significant.

\section{Conclusion}

In this paper, we study the cooperative spectrum sharing between multiple PUs and multiple SUs using the matching theory. We formulate the problem as a twosided matching market, and characterize the market equilibrium systematically. We further study which equilibrium will actually emerge in both complete and incomplete information scenarios. Our study can facilitate the design of large networks, in which players can be split into two different types, interacting with each other. The interaction between players is not restricted within the cooperative spectrum sharing studied in this work, but can be quite general (e.g., spectrum trading). There are some possible directions to extend the results in this paper. An interesting direction is to consider a more general cooperative spectrum sharing protocol where each PU can cooperate with multiple SUs to relay its traffic, and each SU can cooperates with multiple PUs to access their spectrum. In this case, the resulting matching is no longer a one-to-one correspondence between PUs and SUs, but a multiple-to-multiple correspondence between PUs and SUs. 


\section{REFERENCES}

[1] Y. Yuan, P. Bahl, R. Chandra, T. Moscibroda, and Y. Wu. Allocating dynamic time-spectrum blocks in cognitive radio networks. In Proc. of ACM MobiHoc, pages 130-139, 2007.

[2] R. Murty, R. Chandra, T. Moscibroda, and P. Bahl. Senseless: A database-driven white spaces network. In Proc. of IEEE DySPAN, pages 10-21, 2011.

[3] H. Xu and B. Li. Efficient resource allocation with flexible channel cooperation in ofdma cognitive radio networks. In Proc. of IEEE INFOCOM, pages 1-9, 2010.

[4] H. Wu, F. Yang, K. Tan, J. Chen, Q. Zhang, and Z. Zhang. Distributed channel assignment and routing in multiradio multichannel multihop wireless networks. IEEE J. Sel. Areas in Comm., 24(11):1972-1983, 2006.

[5] X. Zhou and H. Zheng. Trust: A general framework for truthful double spectrum auctions. In Proc. IEEE INFOCOM, 2009.

[6] X. Zhou, S. Mettu, H. Zheng, and E.M. Belding. Traffic-driven dynamic spectrum auctions. In Proc. of IEEE SECON, 2008.

[7] X. Wang Q. Zhang M. Dong, G. Sun. Combinatorial auction with time-frequency flexibility in cognitive radio networks. In Proc. of IEEE INFOCOM, 2012.

[8] O. Simeone, I. Stanojev, and et al. Spectrum leasing to cooperating secondary ad hoc networks. IEEE J. Sel. Areas in Comm., 2008.

[9] Y. Han, A. Pandharipande, and S.H. Ting. Cooperative spectrum sharing via controlled amplify-and-forward relaying. In Proc. of IEEE PIMRC, 2008.

[10] J. Zhang and Q. Zhang. Stackelberg game for utility-based cooperative cognitiveradio networks. Proc. ACM MobiHoc, 2009.

[11] H. Wang, L. Gao, and et al. Cooperative spectrum sharing in cognitive radio networks: a game-theoretic approach. In Proc. of IEEE ICC, 2010.

[12] Y. Yan, J. Huang, X. Zhong, and J. Wang. Dynamic spectrum negotiation with asymmetric information. Proc. GameNets, 2011.

[13] L. Duan, L. Gao, and J. Huang. Contract-based cooperative spectrum sharing. In Proc. of IEEE DySPAN, 2011.

[14] S. Bayat, R. Louie, Y. Li, and B. Vucetic. Cognitive radio relay networks with multiple primary and secondary users: Distributed stable matching algorithms for spectrum access. In Proc. of IEEE ICC, 2011.

[15] D. Gale and L.S. Shapley. College admissions and the stability of marriage. The American Mathematical Monthly, 69(1):9-15, 1962.

[16] L.S. Shapley and M. Shubik. The assignment game i: The core. International Journal of Game Theory, (1), 1971.

[17] G. L. Thompson. Computing the core of a market game. Technical report, DTIC Document, 1980.

[18] V.P. Crawford and E.M. Knoer. Job matching with heterogeneous firms and workers. Econometrica: Journal of the Econometric Society, 1981.

[19] A.S. Kelso Jr and V.P. Crawford. Job matching, coalition formation, and gross substitutes. Econometrica: Journal of the Econometric Society, 1982.

[20] A.E. Roth. Misrepresentation and stability in the marriage problem. Journal of Economic Theory, 1984.

[21] F. Kojima and P.A. Pathak. Incentives and stability in large twosided matching markets. The American Economic Review, 2009.

[22] Y. Yi, J. Zhang, Q. Zhang, T. Jiang, and J. Zhang. Cooperative communication-aware spectrum leasing in cognitive radio networks. In Proc. of IEEE DySPAN, 2010.

[23] J.N. Laneman, D.N.C. Tse, and G.W. Wornell. Cooperative diversity in wireless networks: Efficient protocols and outage behavior. IEEE T. on Information Theory, 2004.

[24] D. E. Knuth. Mariages Stables. Les Presses de LUniversite de Montreal, 1976.

[25] V. Krishna. Auction theory. Academic press, 2009.

[26] A. Goldsmith. Wireless communications. Cam. Univ Pr.

[27] H. Wan, J.-F. Diouris, and G. Andrieux, "Time synchronization for cooperative communication in wireless sensor networks," Wireless Personal Communications, 63(4) 977-993, 2002.

[28] Mohsen Nader Tehrani, Mustafa Uysal, and Halim Yanikomeroglu, "Device-to-device communication in 5G cellular networks: challenges, solutions, and future directions," IEEE Communications Magazine, 52(5):86-92, 2014 '

[29] L. Gao, L. Duan, and J. Huang, Tech. Report, [Online] http://jianwei.ie.cuhk.edu.hk/publication/AppendixTwoSided.pd

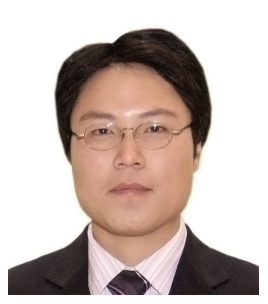

Lin Gao (S'08-M'10) is an Associate Professor in the School of Electronic and Information Engineering, Harbin Institute of Technology (HIT) Shenzhen Graduate School. He received the M.S. and Ph.D. degrees in Electronic Engineering from Shanghai Jiao Tong University (China) in 2006 and 2010, respectively. He worked as a Postdoc Research Fellow at the Chinese University of Hong Kong from 2010 to 2015. His research interests are in the area of network economics and games, with applications in wireless communications, networks, and internet of things.

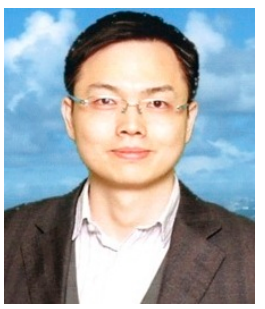

Lingjie Duan (S'09-M'12) is an Assistant Professor with Engineering Systems and Design, Singapore University of Technology and Design (SUTD), Singapore. He received the Ph.D. degree from the Chinese University of Hong Kong, Hong Kong, in 2012. In 2011, he was a Visiting Scholar University of California at Berkeley, CA, USA. He is an Editor of IEEE Communications Surveys and Tutorials, and is a SWAT member in the Editorial Board of IEEE Transactions on Vehicular Technology. He is also a Guest Editor of IEEE Wireless Communications magazine for a special issue about green networking and computing for $5 \mathrm{G}$. His research interests include network economics and game theory, cognitive communications and cooperative networking, energy harvesting wireless communications, and network security. Recently, he served as the Program Co-Chair of the IEEE INFOCOM 2014 GCCCN Workshop, ICCS 2014 special session on Economic Theory and Communication Networks, the Wireless Communication Systems Symposium of the IEEE ICCC 2015, the GCNC Symposium of the IEEE ICNC 2016, and the IEEE INFOCOM 2016 GSNC Workshop. He also served as a technical program committee (TPC) member of many leading conferences in communications and networking (e.g., ACM MobiHoc, IEEE SECON, ICC, GLOBECOM, WCNC, and NetEcon). He was the recipient of the 10th IEEE ComSoc Asia-Pacific Outstanding Young Researcher Award in 2015, the Hong Kong Young Scientist Award (Finalist in Engineering Science track) in 2014, and the CUHK Global Scholarship for Research Excellence in 2011.

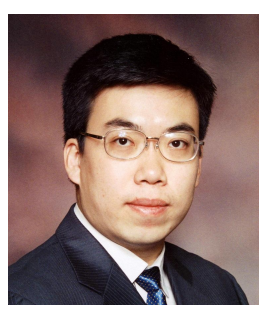

Jianwei Huang (S'01-M'06-SM'11-F'16) is an Associate Professor and Director of the Network Communications and Economics Lab (ncel.ie.cuhk.edu.hk), in the Department of Information Engineering at the Chinese University of Hong Kong. He received the Ph.D. degree from Northwestern University in 2005, and worked as a Postdoc Research Associate in Princeton University during 2005-2007. He is the corecipient of 8 international Best Paper Awards, including IEEE Marconi Prize Paper Award in Wireless Communications in 2011. He has co-authored four books: "Wireless Network Pricing," "Monotonic Optimization in Communication and Networking Systems," "Cognitive Mobile Virtual Network Operator Games, and "Social Cognitive Radio Networks". He has served as an Associate Editor of IEEE Transactions on Cognitive Communications and Networking, IEEE Transactions on Wireless Communications, and IEEE Journal on Selected Areas in Communications - Cognitive Radio Series. He is the Vice Chair of IEEE ComSoc Cognitive Network Technical Committee and the Past Chair of IEEE ComSoc Multimedia Communications Technical Committee. He is a Fellow of IEEE and a Distinguished Lecturer of IEEE Communications Society. 


\section{APPENDIX}

Outline of this appendix:

- A. Strongly Incomplete Information

- B. Illustrations of Equilibria in Simulations

- C. Implementation Issues

- D. Equilibrium Analysis for the Simplified Model

- E. Proof for Theorem 1

- F. Proof for Lemma 3

- G. Proof for Theorem 2

\section{A. Strongly Incomplete Information}

In the main manuscript, we have studied the complete information and incomplete information scenarios. Now we consider the strongly incomplete information scenario, where each PU only knows its own channel and relay channel information, but not $\mathrm{SUs}^{\prime}$ channel information. In this case, each PU does not have enough information to solve the optimization problem (11), even if it knows the reservation utility of its matched SU. Thus, we need to consider not only the mis-representations of SUs, but also the proposing rule with which PUs can calculate the desired offers to SUs.

To this end, we will first design a proposing rule for PUs based on their "guesses" about SUs' information. Then we will study the incentive for SUs to truthfully represent their preferences under the new proposing rule, and characterize the corresponding equilibrium.

The basic idea of the guess-based proposing rule is as follows. First, each PU $m$ guesses the necessary information (called type) about every SU, and proposes an offer aiming at extracting the entire cooperation gain from an SU with the guessed information.20 If it is rejected by an SU, the PU updates its guess about this SU, and propose a new offer (aiming at extracting the entire cooperation gain from the SU with the updated guess).

To capture the key information that PU $m$ wants to know about SUs, we introduce the concept of type for each SU $m$, denoted by $H_{n}^{m}$ and defined as

$$
H_{n}^{m}=2 \cdot \frac{R_{n(m)}-C_{n}}{C_{n} T_{m}} .
$$

Notice that $H_{n}^{m}$ captures all of the key information of SU $n$ (for PU $m$ ). With the type $H_{n}^{m}$, the SU's utility defined in (8) can be rewritten as

$$
\Delta_{n}^{m}=\left(t_{n}^{m} \cdot H_{n}^{m}-p_{n}^{m}\right) \cdot A,
$$

where $A \triangleq \frac{C_{n} T_{m}}{2 \cdot\left(T_{m}+t_{n}^{m}\right)}$ is a constant.

By (16), we can easily find that to ask for a relay power $p_{n}^{m}$ from SU $n$, PU $m$ has to reward an access time no smaller than $t_{n}^{m} \cdot H_{n}^{m}$ to SU $n$ (otherwise SU $n$ will get a negative utility, and thus will never accept the offer). Obviously, PU $m$ is able to derive the optimal proposing rule to SU $n$ from (11) only if it knows the real type

20. Note that we can neither prove the optimality of this proposing rule, nor find another rule always better than this one. Nevertheless, through simulations we can see that the PUs' performances with this proposing rule are very closed to those with optimal proposing rule (11) under weakly incomplete information.
$H_{n}^{m}$. In this scenario, however, PU $m$ cannot obtain the real type $H_{n}^{m}$ of SU $n$, since it does not know the direct channel gain $G_{n(m)}$ of SU $n$.

Let us denote $\widetilde{H}_{n}^{m}$ as the PU $m$ 's guess about the SU $n$ 's real type $H_{n}^{m}$. With this guess $H_{n}^{m}$, PU $m$ aims at extracting all the cooperation gain from $\mathrm{SU} n$, i.e.,

$$
\begin{gathered}
\widetilde{\Pi}_{n}^{m *}=\max _{\left\{p_{n}^{m}, t_{n}^{m}\right\}} \Pi_{n}^{m}\left(p_{n}^{m}, t_{n}^{m}\right) \\
\text { s.t. } \quad p_{n}^{m}=t_{n}^{m} \cdot \widetilde{H}_{n}^{m} .
\end{gathered}
$$

The constraint in 17 shows that (i) if $H_{n}^{m}=\widetilde{H}_{n}^{m}$ (i.e., the guessed SU type is exactly same as the true SU type), then SU $n$ will achieve a zero utility from the PU $m$ 's proposed offer given by (17), and (ii) if $H_{n}^{m}>\widetilde{H}_{n}^{m}\left(\right.$ or $\left.H_{n}^{m}<\widetilde{H}_{n}^{m}\right)$, then SU $n$ can achieve a positive (or negative) utility from the PU $m^{\prime}$ 's proposed offer. Thus, under this guess-based proposing rule, SU $n$ will accept (or reject) the PU $m$ 's offer only if its real type $H_{n}^{m}$ is larger (or smaller) than the PU $m^{\prime}$ s guess $\widetilde{H}_{n}^{m}$. In addition, when accepting the PU's offer (i.e., $H_{n}^{m}>\widetilde{H}_{n}^{m}$ ), SU $n$ 's actual utility is

$$
\begin{aligned}
\widetilde{\Delta}_{n}^{m *} & =\frac{t_{n}^{m *} \cdot\left(R_{n}-C_{n}\right)-p_{n}^{m *} \cdot \frac{C_{n} T_{m}}{2}}{T_{m}+t_{n}^{m *}} \\
& =\frac{C_{n} T_{m}}{2} \cdot \frac{t_{n}^{m *}}{T_{m}+t_{n}^{m *}} \cdot\left(H_{n}^{m}-\widetilde{H}_{n}^{m}\right),
\end{aligned}
$$

where $t_{n}^{m *}$ and $p_{n}^{m *}$ are the optimal solution to (17).

Note that $\widetilde{\Pi}_{n}^{m *}, \widetilde{\Delta}_{n}^{m *}, t_{n}^{m *}$, and $p_{n}^{m *}$ are all functions of $\widetilde{H}_{n}^{m}$. Thus, 17) and (18) define an implicit function between $\widetilde{\Pi}_{n}^{m *}$ and $\widetilde{\Delta}_{n}^{m *}$. Similar to the Utility Transfer Function (UTF) defined in (11), we define a Guessbased Utility Transferring Function (GS-UTF), denoted by $\widetilde{\Pi}_{n}^{m *} \triangleq \widetilde{f}_{n}^{m}\left(\widetilde{\Delta}_{n}^{m *}\right)$. It is easy to check that $\widetilde{\Pi}_{n}^{m *}$ strictly increases with $\widetilde{H}_{n}^{m}$, and $\widetilde{\Delta}_{n}^{m *}$ strictly decreases with $\widetilde{H}_{n}^{m}$. Thus, $\widetilde{f}_{n}^{m}\left(\widetilde{\Delta}_{n}^{m *}\right)$ is a strictly decreasing function of $\widetilde{\Delta}_{n}^{m *}$. Figure 6 in Section 8 illustrates this guess-based utility transferring function.

Notice that all results based on UTF in Section 6, A-D can be directly applied to the model based on GS-UTF here. Specifically, the necessary and sufficient conditions for an equilibrium can be characterized by Theorem 1 (by simply replacing all involved UTF functions into the GS-UTF functions). The optimality of equilibrium can be proved by a similar Lattice theorem in Lemma 3. Note that here the "optimality" refers to the optimal equilibrium restricted within the above guess-based proposing rule, but not the globally optimal equilibrium in all possible proposing rules. As mentioned previously, it is challenging to characterize the globally optimal equilibrium.

Similarly, we can obtain a lower-bound equilibrium (in terms of PUs' utilities) by a "Guess-based Generalized Reversed Deferred Acceptance" procedure (GSGRDAC), which is the same as the G-RDAC except the utility transferring function. The details of the GSGRDAS is skipped due to the space limit. We referred to the equilibrium resulting from the GSG-RDAC as PU-Pessimistic-EQ-GS. Obviously, PU-Pessimistic-EQ-GS 
guarantees the worst-case utility of every PU, with the above guess-based proposing rule, under all possible mis-representations of SUs.

\section{B. Illustrations of Equilibria in Simulations}

Now we illustrate the equilibria (i.e., utility division and relay assignment) in simulations.

\section{(1) Utility Division under Equilibrium}

We first illustrate the utility division (or equivalently, resource exchange) under market equilibria to provide an illustrative impression on the market equilibrium.

Figure 6 illustrates the utility transfer functions (UTF) and the associated equilibrium utility division in the network with (i) one PU $\{m\}$ and two SUs $\left\{n_{1}, n_{2}\right\}$ (left figure) and (ii) two PUs $\left\{m_{1}, m_{2}\right\}$ and one SU $\{n\}$ (right figure). Recall that the UTF function represents how a PU's maximal utility changes with the utility of the matched SU. In the left figure, we choose the symmetric topology for the PU and two SUs (with $G_{m}^{2}=-110 \mathrm{dBm}$, $\left.G_{n(m)}^{2}=G_{m, n}^{2}=G_{n, m}^{2}=-90 \mathrm{dBm}, n \in\left\{n_{1}, n_{2}\right\}\right)$ and different energy costs for two SUs (with $C_{n_{1}}=1$ and $C_{n_{2}}=2$ ). In this case, PU $m$ is matched to SU $n_{1}$ under the market equilibrium. In the right figure, we choose different topologies for the SU and two PUs (with $G_{m_{1}}^{2}=$ $-110 \mathrm{dBm}, G_{m_{2}}^{2}=-100 \mathrm{dBm}, G_{n(m)}^{2}=G_{m, n}^{2}=G_{n, m}^{2}=$ $\left.-90 \mathrm{dBm}, m \in\left\{m_{1}, m_{2}\right\}\right)$. In this case, $\mathrm{SU} n$ is matched to PU $m_{1}$ under the market equilibrium. In both figures, the equilibrium utility division among the matched PU and SU is illustrated by the black bold line (between the circle and the square). More specifically, the circle denotes the utility division under the Pareto-optimal equilibrium for PUs (PU-Optimal-EQ), and the square denotes the utility division under the Robust equilibrium for PUs (PU-Robust-EQ). More detailed explanations for these equilibria are given below.

(1) PU-Optimal-EQ in the left of Figure 6 The PUOptimal-EQ can be achieved by offering the lowest acceptable utility (i.e., zero in this example) to SU $n_{1}$. Obviously, the PU achieves its maximum utility under PU-Optimal-EQ among all equilibria.

(2) PU-Robust-EQ in the left of Figure 66 The PURobust-EQ can be achieved by offering the highest achievable utility (i.e., 0.5 in this example) to SU $n_{1}$. Note that due to the competition among SUs, SU $n_{1}$ cannot requesting a utility higher than 0.5 from the PU (which will leave the PU a utility lower than 0.8), otherwise, $\mathrm{SU} n_{2}$ can successfully pair with the PU by requesting a utility slightly higher than zero (which will leave the PU a utility of 0.8 ). This implies that the PU is guaranteed to obtain a utility of 0.8 (i.e., that under PU-Robust-EQ).

(3) PU-Optimal-EQ in the right of Figure 6. The PUOptimal-EQ can be achieved when PU $m_{1}$ offers the lowest acceptable utility (i.e., 1.7 in this example) to SU $n$. Note that due to the competition among PUs, PU $m_{1}$ cannot offer a utility lower than 1.7 to SU $n$, otherwise, PU $m_{2}$ can offer a utility of 1.7 to SU $n$ such that SU $n$

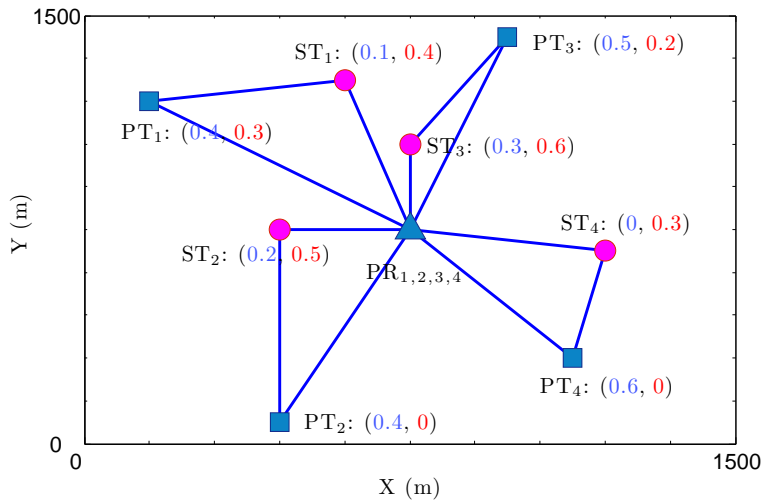

Fig. 7. Relay Assignment under Market Equilibrium. Each $\mathrm{PU} i$ is matched to SU $i$. The value associated with each $\mathrm{PT}_{i}$ (or $\mathrm{ST}_{i}$ ) denote $\mathrm{PU} i$ 's (or $\mathrm{SU} i$ 's) utilities under PUOptimal-EQ (blue) and PU-Robust-EQ (red), respectively.

accepts the offer of PU $m_{2}$. Similarly, PU $m_{1}$ achieves its maximum utility under the PU-Optimal-EQ among all equilibria ${ }^{21}$

(4) PU-Robust-EQ in the right of Figure 65. The PURobust-EQ can be achieved when PU $m_{1}$ offers the highest achievable utility (i.e., 2.2 in this example) to SU $n$. Note that PU $m_{1}$ will never offer a utility higher than 2.2 to SU $n$, otherwise, it will achieve a negative utility. Therefore, the PU-Robust-EQ provides PU $m_{1}$ a guaranteed utility of zero.

From Figure 6, we can also see that the PUs' and SUs' equilibrium utilities are greatly affected by the numbers of PUs and SUs. Specifically, when the number of PUs is smaller than that of SUs (left figure), the PU is likely to achieve a large utility under the market equilibrium, benefiting from the competition of SUs. When the number of PUs is larger than that of SUs (right figure), the PUs are likely to achieve small utilities under the market equilibrium, suffering from the competition of PUs. Later in Figure 5 we will show this observation more explicitly.

\section{(2) Relay Assignment under Equilibrium}

Now we illustrate the relay assignment under the market equilibrium. To provide a clear impression, we simulate a network with 4 PUs and 4 SUs shown in Figure 7. where primary receivers (PRs) are co-located at the middle of the area (denoted by the trangle), and primary transmitters (PTs) are located close to the edges of the area (denoted by squares). We use the default network setting except the channel gains, which are derived by the free-space path loss model in [26].

Figure 7 illustrates the network topology and the associated relay assignment (i.e., PU-SU matching) under the market equilibrium. In this example, SU $i$ is assigned to relay for PU $i, \forall i=1,2,3,4$, under the market equilibrium. Note that PUs' and SUs' utilities are different under different market equilibria. The values associated with each $\mathrm{PT}_{i}$ (or $\mathrm{ST}_{i}$ ) denote $\mathrm{PU} i$ 's (or $\mathrm{SU}$

21. Note PU $m_{2}$ achieves a zero utility under the PU-Optimal-EQ. 

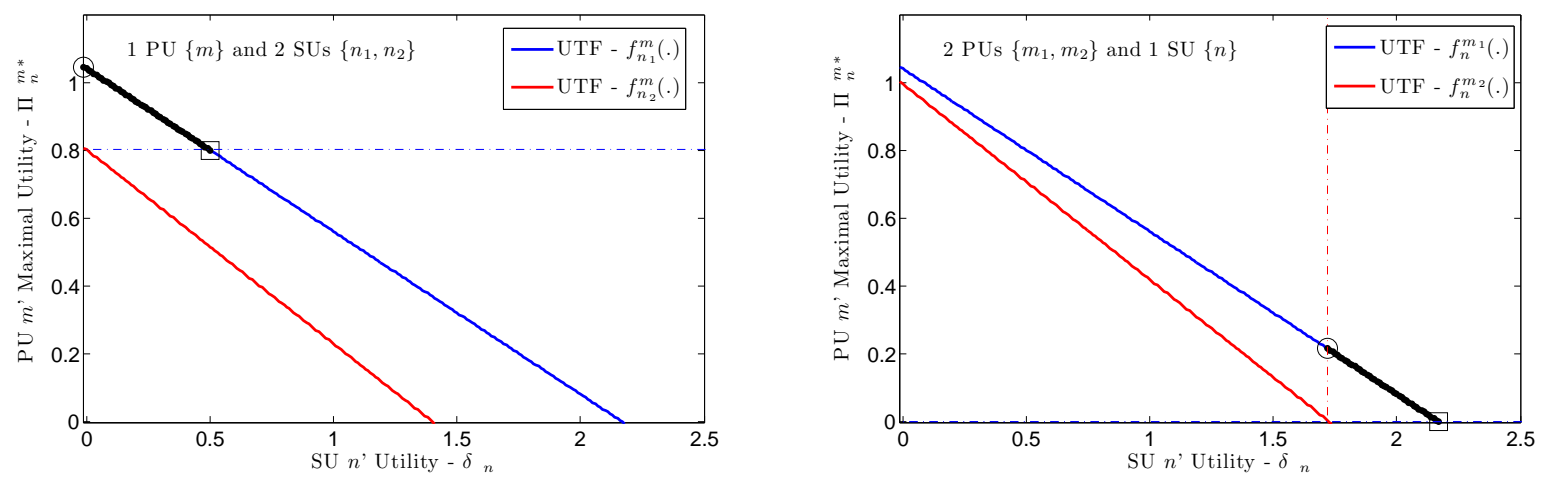

Fig. 6. Utility Division under Market Equilibrium. Left: one PU $\{m\}$ and two SUs $\left\{n_{1}, n_{2}\right\}$. PU $m$ is matched to SU $n_{1}$ under the market equilibrium. Right: two PUs $\left\{m_{1}, m_{2}\right\}$ and one SU $\{n\}$. SU $n$ is matched to PU $m_{1}$ under the market equilibrium. The equilibrium utility division among the matched PU and SU is illustrated by the black bold line.

$i^{\prime}$ s) utilities under the PU-Optimal-EQ (blue) and the PU-Robust-EQ (red), respectively. Specifically, under the PU-Optimal-EQ, PUs' utilities are $\{0.4,0.4,0.5,0.6\}$, and SUs' utilities are $\{0.1,0.2,0.3,0\}$; Under the PU-RobustEQ, PUs' utilities are $\{0.3,0,0.2,0\}$, and $\mathrm{SUs}^{\prime}$ utilities are $\{0.4,0.5,0.6,0.3\}$. Detailed discussions about such a utility difference are given below.

(1) PU 4 and SU 4: PU 4 and SU 4 are far apart from other users, and their interaction will be slightly affected by others' interactions. Thus, under the PU-Optimal-EQ, SU 4 will achieve the lowest acceptable utility (i.e., 0 ). Under the PU-Robust-EQ, SU 4 will achieve the highest acceptable utility (i.e., 0.3), and PU 4 can only achieve a zero utility.

(2) PUs 1 and 3: PU 1 is close to SUs $\{1,2,3\}$, and can potentially cooperate with these SUs. Thus, SUs $\{1,2,3\}$ will compete with each for PU 1 . Similar to the left figure of Figure 6 the PU can achieve a high utility (i.e., 0.3) under the (worst-case) robust equilibrium PU-Robust$E Q$, benefiting from the competition of SUs. Similarly, PU 3 can potentially cooperate with multiple SUs $\{1,3\}$, and thus will also achieve a high utility (i.e., 0.2 ) under the PU-Robust-EQ.

\section{Implementation Issues}

In this subsection, we briefly discuss the implementation issues of the proposed cooperative sharing scheme.

We first discuss how PUs obtain information in different information scenarios. As shown in Section 4.3 . we consider two information scenarios: complete and incomplete information. First, complete information is an ideal benchmark (though not practical) case, where PUs know everything about the network. Second, incomplete information is a more practical case, where each PU knows only the local information, including its own channel gain, its relay channel gains (with neighboring SUs), and the neighboring SUs' channel gains. The first class of local information can be easily obtained via measuring the average Received Signal Strength (RSS) from the PU's transmitter to receiver. The second class of local information can be obtained via measuring the average RSS from SUs's transmitters to the PU's transmitter and receiver, respectively. The third class of local information (i.e., SUs' channel gains), however, cannot be measured by the PU directly. Nevertheless, by measuring the average RSS from a SU's transmitter to the PU's transmitter and receiver jointly, the PU can estimate the SU transmitter's location (i.e., obtaining two candidate points); with the similar method, the PU can estimate the SU receiver's location (i.e., obtaining two candidate points). Hence, there are at most four possibilities for an SU's topology. With certain additional knowledge (e.g., historical information or SU report), the PU can derive the exact topology of the SU, and further calculate the SU's average channel gain ${ }^{22}$

We then discuss the time synchronization, which is a very important implementation issue. In the existing literature of cooperative communications, researchers have proposed many techniques to address this issue (see, e.g., [27]). In our model, we would like to clarify that we do not require all PUs and SUs to be fully synchronized during the whole interaction period. As in many existing literature (e.g., [8]-[14]), we assume that there exists a common control channel for the necessary communications and interactions between PUs and SUs. Namely, PUs and SUs transmit all of the control signals (e.g., beacon, PU proposal, and SU response) on the common control channel using certain multiple access method such as CDMA, and continuously monitor the common control channel to decode the desired control signals. Hence, PUs and SUs can interact (on the common control channel) in the asynchronous manner during the matching period (i.e., in the processing of Algorithms 2 and 3); when a stable matching is reached (i.e., after the convergence of Algorithms 2 and 3), each pair of matched PU and SU then operate (on the PU's operating channel) in the synchronous manner during

22. It is important to note that such an estimation is based on assumption that there is no deep fading or shadowing between the SU's transmitter and receiver, hence the channel gain is determined by the transmission distance only. Otherwise, the PU may not be able to estimate the SU's own channel gain correctly. In that case, the estimation will be based on the SU's report directly. 
their cooperative transmission period (Phases I and II) and secondary transmission period (Phase III). Such a synchronization (among a pair of matched PU and SU in Phases I, II, and III) can be achieved using different methods in the existing cooperative communication literature (e.g., [27]).

\section{Equilibrium Analysis for the Simplified Model}

Now we provide the complete equilibrium analysis for the simplified model.

\section{(1) Common \& Conflicting Interests on Equilibrium}

By Example 1. we can find that there exists a unique Pareto-optimal equilibrium for PUs: $\mu^{a}$, where every PU achieves a no worse utility than in any other equilibrium. Similarly, there exists a unique Pareto-optimal equilibrium for SUs: $\mu^{c}$, where every SU achieves a no worse utility than in any other equilibrium. Lemma 5 shows that this observation is generally applicable.

Lemma 5 (Optimality): There always exists a unique Pareto-optimal equilibrium for PUs, where every PU achieves its maximum utility among all equilibria. Similarly, there always exists a unique Pareto-optimal equilibrium for SUs, where every SU achieves its maximum utility among all equilibria.

The following lemma further shows that among any two equilibria, the equilibrium that is better for all users on one side is always worse for all users on the other side.

Lemma 6 (Conflicting Interest): For any equilibria $\mu$ and $\mu^{\prime}$, all PUs prefer $\mu$ to $\mu^{\prime}$, if and only if all SUs prefer $\mu^{\prime}$ to $\mu$.

Lemmas 5 and 6 show that users on the same side have a common interest and users on the opposite sides have conflicting interests, regarding the set of equilibria ${ }^{23}$

\section{(2) Equilibrium under Complete Information}

Now we study which equilibrium will actually emerge (in the PU-proposal market) under complete information. In this case, any SU's misrepresentation (of its preference list) is not allowed, as PUs know the whole network information and thus know the complete preference lists of SUs.

We first introduce the "deferred acceptance" (DAC) algorithm in [15], and then show in Lemma 7 the DAC algorithm converges to the Pareto-optimal equilibrium for PUs (PU-Optimal-EQ).

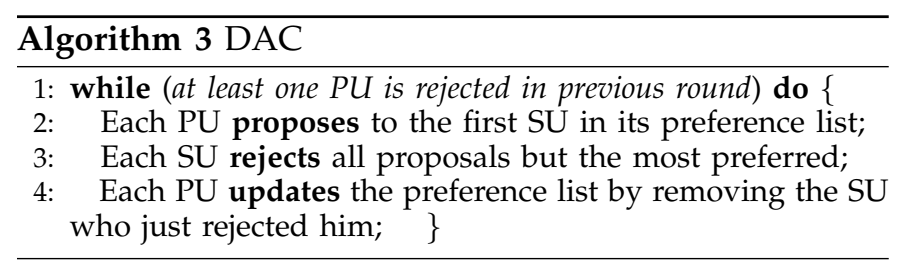

23. This observation was first discovered by Gale and Shapley in [15]. The detailed proof can also be referred to [15].
Lemma 7: The DAC algorithm converges the Paretooptimal equilibrium for PUs, i.e., PU-Optimal-EQ.

Notice that every PU can achieve a better utility under the PU-Optimal-EQ than under any other equilibrium. That is, there does not exist another equilibrium, in which at least one PU achieves a higher utility than in the PU-Optimal-EQ. Thus, all PUs are willing to follow the proposing rule in the DAC algorithm in order to achieve the PU-Optimal-EQ. This implies that the PU-Optimal-EQ is the only equilibrium that will emerge under complete information.

\section{(3) Equilibrium under Incomplete Information}

Now let us consider the incomplete information scenario, where PUs know their local network information only, and thus cannot know the complete preference lists of SUs. Therefore, it is possible for SUs to misrepresent their preference lists to seek more utilities. We show this by the following example.

Example 3: Consider the same model in Example 1. except that here each PU does not know SUs' preference lists. Suppose SU $n_{1}$ misrepresents its preferences by $Q\left(n_{1}\right)=\left\{m_{2}, m_{3}\right\}$. Then it is easy to check that the DAC algorithm will lead to the equilibrium $\mu^{b}$. Furthermore, if SU $n_{1}$ misrepresents its preferences by $Q\left(n_{1}\right)=\left\{m_{2}\right\}$, then the DAC algorithm will lead to the Pareto-optimal equilibrium $\mu^{c}$ for SUs. In both misrepresentations, SU $n_{1}$ achieves a higher utility than truth-telling.

By the above example, we can see that different misrepresentation behaviors of SUs may lead to different market equilibria. Unfortunately, characterizing all misrepresentation behaviors of SUs in the incomplete information scenario is an NP-hard problem. Hence, it is difficult to characterize which specific equilibrium will actually emerge in the incomplete information scenario, due to the uncertainty of SU misrepresentation. To this end, we study the worst-case equilibrium for PUs, and characterize a robust equilibrium for PUs (PU-Robust$E Q)$, which gives every PU a guaranteed utility under any possible misrepresentations of SUs.

Specifically, the robust equilibrium PU-Robust-EQ can be achieved by a "reversed deferred acceptance" (RDAC) algorithm as in [15], which is same as the DAC algorithm except that it reverses the roles of PUs and SUs in proposing offers. That is, in the RDAC algorithm, each SU proposes to its most preferred PU among those who have not yet rejected him, and each PU accepts the most preferred proposal and rejects the others. The details of the RDAC algorithm are skipped due to space limit. By the symmetry between two algorithms, the RDAC algorithm converges to the Pareto-optimal equilibrium for SUs, which, by Lemma 6 , is the worst-case equilibrium for PUs. Therefore, every PU is guaranteed to achieve a utility no worse than that in this worst-case equilibrium, under any possible misrepresentations of SUs. In this sense, we refer to this worst-case equilibrium for PUs as the robust equilibrium for PUs. 


\section{E. Proof for Theorem 1}

We have already shown the necessity by the above analysis. Next we prove the sufficiency.

We first show that such a matching does not violate any PU or SU's IR condition. This is because each SU $n$ gets a utility $\delta_{n} \geq \underline{\delta}_{n} \geq 0$ and each PU $m$ gets a utility $f_{n}^{\mu_{n}}\left(\delta_{n}\right) \geq f_{n}^{\mu_{n}}\left(\bar{\delta}_{n}\right) \geq f_{n}^{\mu_{n}}\left(g_{n}^{\mu_{n}}(0)\right)=0$. By (11), we can further show that each pair of matched PU $\mu_{n}$ and SU $n$ have no incentive to change $\delta_{n}$, otherwise at least one user will lose certain utility (since an increase of one's utility will lead to a decrease of another's utility).

We then show that no PU or SU will deviate from the current matching by choosing a different partner. We prove this by contradiction. Suppose that both PU $\mu_{n}$ (who is currently matched to SU $n$ ) and SU $k \neq n$ have incentives to break the matching by pairing up with each other. Then there must exist a value $\delta_{k}^{\prime}$ such that (i) $\delta_{k}^{\prime}>$ $\delta_{k}$ (i.e., SU $k$ achieves a larger utility with PU $\mu_{n}$ ) and (ii) $f_{k}^{\mu_{n}}\left(\delta_{k}^{\prime}\right)>f_{n}^{\mu_{n}}\left(\delta_{n}\right)$ (i.e., PU $\mu_{n}$ achieves a larger utility with SU $k$ ). On the other hand, we have

$$
f_{n}^{\mu_{n}}\left(\delta_{n}\right) \geq f_{k}^{\mu_{n}}\left(\delta_{k}\right)>f_{k}^{\mu_{n}}\left(\delta_{k}^{\prime}\right),
$$

where the first inequality follows directly from $\delta_{n} \leq \bar{\delta}_{n}$, and the second inequality follows from the assumption $\delta_{k}^{\prime}>\delta_{k}$. This leads to a contradiction.

\section{F. Proof for Lemma 3}

To prove $\left\{\left(\mu_{n}, \delta_{n}^{X}\right), \forall n\right\}$ is an equilibrium, we only need to show that $\delta_{n}^{X}$ satisfies the conditions in Theorem 1 . By Theorem 1, we have: for all $n \in \mathcal{N}$,

$$
\underline{\delta}_{n}^{i} \leq \delta_{n}^{i} \leq \bar{\delta}_{n}^{i}, \quad i=I, I I .
$$

Without loss of generality, we consider an arbitrary matching pair, say, PU $\mu_{n}$ and SU $n$. Suppose $\delta_{n}^{I} \leq \delta_{n}^{I I}$. Then $\delta_{n}^{X}=\min \left(\delta_{n}^{I}, \delta_{n}^{I I}\right)=\delta_{n}^{I}$. On one hand,

$$
\begin{aligned}
\delta_{n}^{X}=\delta_{n}^{I} \geq \underline{\delta}_{n}^{I} & =\max _{m \neq \mu_{n}}\left\{g_{n}^{m}\left(f_{\mu_{m}}^{m}\left(\delta_{\mu_{m}}^{I}\right)\right), 0\right\} \\
& \geq \max _{m \neq \mu_{n}}\left\{g_{n}^{m}\left(f_{\mu_{m}}^{m}\left(\delta_{\mu_{m}}^{X}\right)\right), 0\right\} \triangleq \underline{\delta}_{n}^{X} .
\end{aligned}
$$

The second line follows because $g_{n}^{m}\left(f_{\mu_{m}}^{m}(\cdot)\right)$ is an increasing function, and $\delta_{\mu_{m}}^{X} \leq \delta_{\mu_{m}}^{I}, \forall \mu_{m}$. On the other hand,

$$
\begin{aligned}
\delta_{n}^{X} & =\min \left\{\delta_{n}^{I}, \delta_{n}^{I I}\right\} \leq \min \left\{\bar{\delta}_{n}^{I}, \bar{\delta}_{n}^{I I}\right\} \\
& =\min _{k \neq n}\left\{g_{n}^{\mu_{n}}\left(f_{k}^{\mu_{n}}\left(\delta_{k}^{I}\right)\right), g_{n}^{\mu_{n}}\left(f_{k}^{\mu_{n}}\left(\delta_{k}^{I I}\right)\right), g_{n}^{\mu_{n}}(0)\right\} \\
& =\min _{k \neq n}\left\{g_{n}^{\mu_{n}}\left(f_{k}^{\mu_{n}}\left(\min \left(\delta_{k}^{I}, \delta_{k}^{I I}\right)\right)\right), g_{n}^{\mu_{n}}(0)\right\} \triangleq \bar{\delta}_{n}^{X} .
\end{aligned}
$$

The last line follows because $h(\cdot) \triangleq g_{n}^{\mu_{n}}\left(f_{k}^{\mu_{n}}(\cdot)\right)$ is an increasing function, thus $\min (h(x), h(y))=h(\min (x, y))$.

\section{G. Proof for Theorem 2}

The existence and uniqueness of PU-optimal equilibrium can be easily proved by iteratively applying Lemma 3 on any two equilibria. Next we prove that the PU-optimal equilibrium $\left\{\left(\mu_{n}, \delta_{n}^{*}\right), \forall n\right\}$ satisfies the conditions: $\delta_{n}^{*}=$ $\underline{\delta}_{n}^{*}, \forall n$. If not, there must exist some $n$ such that $\delta_{n}^{*}>\underline{\delta}_{n}^{*}$, and $\delta_{n}^{*}$ can be further reduced without affecting the IC and IR conditions for equilibrium. Obviously, this violates the optimality of $\left\{\left(\mu_{n}, \delta_{n}^{*}\right), \forall n\right\}$. 